Research Paper

\title{
Near Infrared Fluorescent Nanoparticles Derived from Hyaluronic Acid Improve Tumor Contrast for Image-Guided Surgery
}

\author{
Tanner K. Hill1, Sneha S. Kelkar², Nicholas E. Wojtynek ${ }^{3}$, Joshua J. Souchek¹, William M. Payne ${ }^{1}$, Kristina \\ Stumpf ${ }^{4}$, Frank C. Marini ${ }^{4,5}$, Aaron M. Mohs ${ }^{1,3}{ }^{\boxplus}$ \\ 1. Department of Pharmaceutical Sciences, University of Nebraska Medical Center, Omaha, NE 68198, United States; \\ 2. Department of Plastic and Reconstructive Surgery, Wake Forest University Health Sciences, Winston-Salem, NC 27157, United States; \\ 3. Fred and Pamela Buffett Cancer Center, University of Nebraska Medical Center, Omaha, NE 68198, United States; \\ 4. Wake Forest Institute for Regenerative Medicine, Wake Forest University Health Sciences, Winston-Salem, NC 27157, United States; \\ 5. Department of Cancer Biology, Wake Forest University Health Sciences, Winston-Salem, NC 27157, United States. \\ $\triangle$ Corresponding author: Aaron M. Mohs, PhD. Department of Pharmaceutical Sciences, University of Nebraska Medical Center, Eppley Cancer Institute, Room 4011, \\ Omaha, NE 68198-6025. Email: aaron.mohs@unmc.edu.
}

(C) Ivyspring International Publisher. Reproduction is permitted for personal, noncommercial use, provided that the article is in whole, unmodified, and properly cited. See http://ivyspring.com/terms for terms and conditions.

Received: 2016.06.17; Accepted: 2016.09.13; Published: 2016.10.01

\begin{abstract}
Tumor tissue that remains undetected at the primary surgical site can cause tumor recurrence, repeat surgery, and treatment strategy alterations that impose a significant patient and healthcare burden. Intraoperative near infrared fluorescence (NIRF) imaging is one potential method to identify remaining tumor by visualization of NIR fluorophores that are preferentially localized to the tumor. This requires development of fluorophores that consistently identify tumor tissue in different patients and tumor types. In this study we examined a panel of NIRF contrast agents consisting of polymeric nanoparticle (NP) formulations derived from hyaluronic acid (HA), with either physically entrapped indocyanine green (ICG) or covalently conjugated Cy7.5. Using orthotopic human breast cancer MDA-MB-231 xenografts in nude mice we identified two lead formulations. One, NanolCGPBA, with physicochemically entrapped ICG, showed 2.3-fold greater tumor contrast than ICG alone at $24 \mathrm{~h}(\mathrm{p}<0.01)$, and another, NanoCy7.5100-H, with covalently conjugated Cy7.5, showed 74-fold greater tumor contrast than Cy7.5 alone at $24 \mathrm{~h}(\mathrm{p}<0.0001)$. These two lead formulations were then tested in immune competent BALB/c mice bearing orthotopic 4T1 breast cancer tumors. NanolCGPBA showed 2.2-fold greater contrast than ICG alone ( $p<0.0001$ ), and NanoCy7.5100-H showed 14.8-fold greater contrast than Cy7.5 alone ( $\mathrm{p}<$ $0.0001)$. Furthermore, both NanolCGPBA and NanoCy7.5100-H provided strong tumor enhancement using image-guided surgery in mice bearing 4T1 tumors. These studies demonstrate the efficacy of a panel of HA-derived NPs in delineating tumors in vivo, and identifies promising formulations that can be used for future in vivo tumor removal efficacy studies.
\end{abstract}

Key words: near infrared fluorescence

\section{Introduction}

Surgery remains one of the most common and effective treatment options for a wide range of tumor types and plays a pivotal role for curative treatment of early stage cancer [1]. Detecting unresected tumor tissue at the site of the primary tumor, so-called positive margins (PMs), remains a substantial concern for surgeons and patients since PMs are reported to occur in $20-40 \%$ of patients receiving breast conserving surgery $[2,3]$. Even among patients that have an additional four to five margin specimens removed during surgery, PM rates are as high as $14.9 \%$ [3]. PMs can result in local and distant recurrence, which requires additional treatment, and are also associated with lower disease-specific survival [2-6]. Thus, improving methods to detect PMs and loco-regional malignant lesions are necessary to improve patient outcome.

Preoperative image modalities such as computed 
tomography, magnetic resonance imaging, and positron emission tomography offer an abundance of information needed for diagnosis and planning [7]. However, these modalities are generally not feasible for real time detection of tumor tissue in the operating room. The most commonly used modalities for real-time tumor detection in the operating room are visual and tactile feedback from the surgeons themselves [8]. There has recently been increased investigations in the field of NIRF image-guided surgery (IGS) as a means to detect diseased tissue during surgery $[9,10]$. NIRF imaging offers a number of advantages. The NIR range, wavelengths of approximately $700-1000 \mathrm{~nm}$, is non-ionizing and thus relatively safe to use for both patient and caregiver [11-13]. NIRF imaging also takes advantage of the "NIR window", where the tissue absorption of light by water and hemoglobin is minimized compared to scattering, allowing for imaging or detection by NIRF up to several millimeters deep $[13,14]$. Synthesis of novel NIR fluorophores, conjugates, and nanoparticles (NPs) may improve delivery to specific tissue types by actively targeting cell-specific moieties or passive targeting by enhanced permeability and retention (EPR) [15].

Several studies have demonstrated the clinical potential for intraoperative image guidance during tumor surgery with the explicit purpose of identifying target tissues such as tumors and sentinel lymph nodes (SLNs) [9,16-20]. The majority of clinical studies have utilized ICG, methylene blue, and 5 -aminolevulinic acid [9]. Most common among these is the NIR fluorophore ICG $\left(\lambda_{\text {ex }} \sim 800 \mathrm{~nm}, \lambda_{\text {em }} \sim 825\right.$ $\mathrm{nm})$, which is both FDA and EMA approved [21]. ICG has been tested in clinics for SLN mapping and for tumor imaging $[9,21]$. Additional NIR fluorophores optimized for active targeting of target tissues are currently under clinical investigation $[22,23]$.

Preclinical studies have been performed to examine targeted NIR dyes and NPs for tumor imaging, such as integrin-targeted cRGD-ZW800-1 and NPs that contain dyes, such as Cy5.5, Cy7.5, and ICG [24-27]. Of interest here are dyes either physicochemically entrapped or directly conjugated to hyaluronic acid (HA). HA is composed of repeating units of $\beta(1,4)$ D-glucuronic acid and $\beta(1,3)$ $\mathrm{N}$-acetyl-D-glucosamine, and naturally occurs in the umbilical cord, synovial fluid, and vitreous body of the eye [28]. HA has been used to form a variety of polymeric NPs by conjugation of hydrophobic moieties to the hydrophilic HA polymer backbone, which drives self-assembly $[25-27,29,30]$. Targeting to CD44 and passive accumulation by EPR may also improve HA-based NP delivery to difficult-to-target tumors such as triple negative breast cancer (TNBC), which lack expression of estrogen receptor, progesterone receptor, and HER2 [31].

We report here on the in vivo imaging evaluation of a panel of eight HA-derived NIRF NPs that were chosen based on initial studies that used physicochemically entrapped ICG [26] or covalently conjugated Cy7.5 [27]. As shown in Figure 1A the HA-derived NPs used in this study consisted of two "NanoICG" formulations, which utilized $10 \mathrm{kDa} H A$ polymers with physically entrapped ICG, and six "NanoCy7.5" formulations, which used HA polymers that were chemically conjugated to Cy7.5 (three with $10 \mathrm{kDa} \mathrm{HA}$, and three with $100 \mathrm{kDa}$ HA polymers). The resulting NPs were delivered to nude mice bearing the TNBC tumor model MDA-MB-231, and examined at 4, 24, and $72 \mathrm{~h}$ (Figure 1B). Biodistribution, tumor contrast, and imaging depth using simulated breast tissue were analyzed. Based on these results, promising NanoICG and NanoCy7.5 formulations were tested in immune competent BALB/c mice bearing $4 \mathrm{~T} 1$ tumors as a second TNBC model [32,33].

\section{Materials and Methods Materials}

HA was purchased from LifeCore Biomedical (Chaska, MN). 1-ethyl-3-(3-dimethylaminopropyl) carbodiimide (EDC) N-hydroxysuccinimide (NHS), $\mathrm{N}, \mathrm{N}$-dimethylformamide (DMF), dimethyl sulfoxide (DMSO), methanol, dialysis membranes (3,500 Da MWCO and 6,000-8,000 Da MWCO), gelatin powder N.F. grade, and TRIS-HCl were purchased from Fisher Scientific. 5 $\beta$-cholanic acid, 1-pyrenebutyric acid, sodium azide, dried erythrocytes, 1,3-diaminopropane, and ICG were purchased from Sigma Aldrich (St. Louis, MO). Ethanol was purchased from the Warner-Graham Company (Cockeysville, MD) or the General Supply at the University of Nebraska Medical Center. Cy7.5-amine was purchased from Lumiprobe Corporation (Hallandale Beach, FL). All the reagents were used without further purification unless otherwise specified. Desalting PD10 columns were purchased from GE Healthcare. MEM, fetal bovine serum, phosphate buffered saline, and penicillin/ streptomycin were obtained from Cell and Viral Vector lab at Wake Forest Health Sciences or from the General Supply at the University of Nebraska Medical Center. All water was obtained from a Barnstead NANOpure Diamond or Barnstead GenPure system (Thermo Scientific; Waltham, MA) producing 18.2 M $\Omega$ water. Human breast cancer cell line MDA-MB 231 and mouse breast cancer 4T1 were obtained from ATCC (Manassas, VA). 


\section{Nanoparticle Synthesis}

ICG-loaded and Cy7.5-conjugated NPs were synthesized as previously described $[26,27,34]$. Briefly, aminopropyl-1-pyrenebutanamide (PBA) and aminopropyl-5 $\beta$-cholanamide $(5 \beta C A)$ were synthesized from 1-pyrenebutyric acid and $5 \beta$-cholanic acid by first refluxing in methanol at $60^{\circ} \mathrm{C}$ for six hours, followed by purification and refluxing in 1,3-diaminopropane at $135{ }^{\circ} \mathrm{C}$ for six hours. $5 \beta C A-H A$ conjugates were synthesized with $10 \mathrm{kDa}$ or $100 \mathrm{kDa} H A$ polymers followed by conjugation with $5 \beta C A$ with 10.4 or $25.7(10 \mathrm{kDa})$ or $32.1(100 \mathrm{kDa})$ wt $\%$ by first dissolving HA 50:50 $\mathrm{H}_{2} \mathrm{O}: \mathrm{DMF}$ containing 10-fold molar excess of EDC/NHS, followed by dropwise addition of 5 $\beta \mathrm{CA}$ and stirring for $24 \mathrm{~h}$. PBA-HA conjugates were synthesized and purified similarly using $10 \mathrm{kDa} \mathrm{HA}$ and $10 \mathrm{wt} \%$ PBA. Each polymer conjugate was purified by dialysis in 50:50 $\mathrm{H}_{2} \mathrm{O}: \mathrm{EtOH}$ for $24 \mathrm{~h}$ followed by dialysis in pure $\mathrm{H}_{2} \mathrm{O}$ for an additional $48 \mathrm{~h}$. Cy7.5-amine was conjugated to HA or amphiphilic HA using EDC/NHS chemistry [27], while ICG was physically loaded as described previously [26]. All NPs were then frozen, lyophilized, and stored at $-20{ }^{\circ} \mathrm{C}$.
NanoICG conjugates with PBA and 5 $\beta C A$ as the hydrophobic moiety are termed, NanoICGPBA and NanoICG $_{5 \beta C A}$, respectively. NanoCy7.5 conjugates are labeled with subscripts identifying the molecular weight used, NanoCy7.510 for $10 \mathrm{kDa}$ or NanoCy7.5100 for $100 \mathrm{kDa}$, followed by the $5 \beta \mathrm{CA}$ conjugation content: none " $\varnothing$ ", low " $\mathrm{L}$ ", or high " $\mathrm{H}$ ", as introduced by Kelkar, et al [27]. For example, NanoCy7.5 composed of $100 \mathrm{kDa}$ HA with high 5 $\beta \mathrm{CA}$ content is identified as NanoCy7.5100-H. Nanoparticle hydrodynamic diameter histograms have previously been reported [26,27] and are summarized here: NanoICG $_{\text {pвA }}=40.1 \mathrm{~nm} ;$ NanoICG $_{5 \beta C A}=71.8 \mathrm{~nm}$; NanoCy7.510- $\varnothing=44.4 \mathrm{~nm}$; NanoCy7.510-L $=111.6 \mathrm{~nm}$; NanoCy7.510-H $=52.4 \mathrm{~nm}$; NanoCy7.5100-ø $=206.8 \mathrm{~nm}$; NanoCy7.5100-L $=590.2$ nm; NanoCy7.5 $5_{100-\mathrm{H}}=444.8$ $\mathrm{nm}$. For in vivo studies, fluorophore dose was determined by dissolving contrast agents in 50:50 $\mathrm{H}_{2} \mathrm{O}$ :DMSO and measuring the absorbance spectra relative to a standard curve. An in vitro nanoparticle stability study at physiological levels of albumin is described in supplemental materials and Supplemental Figure 1.

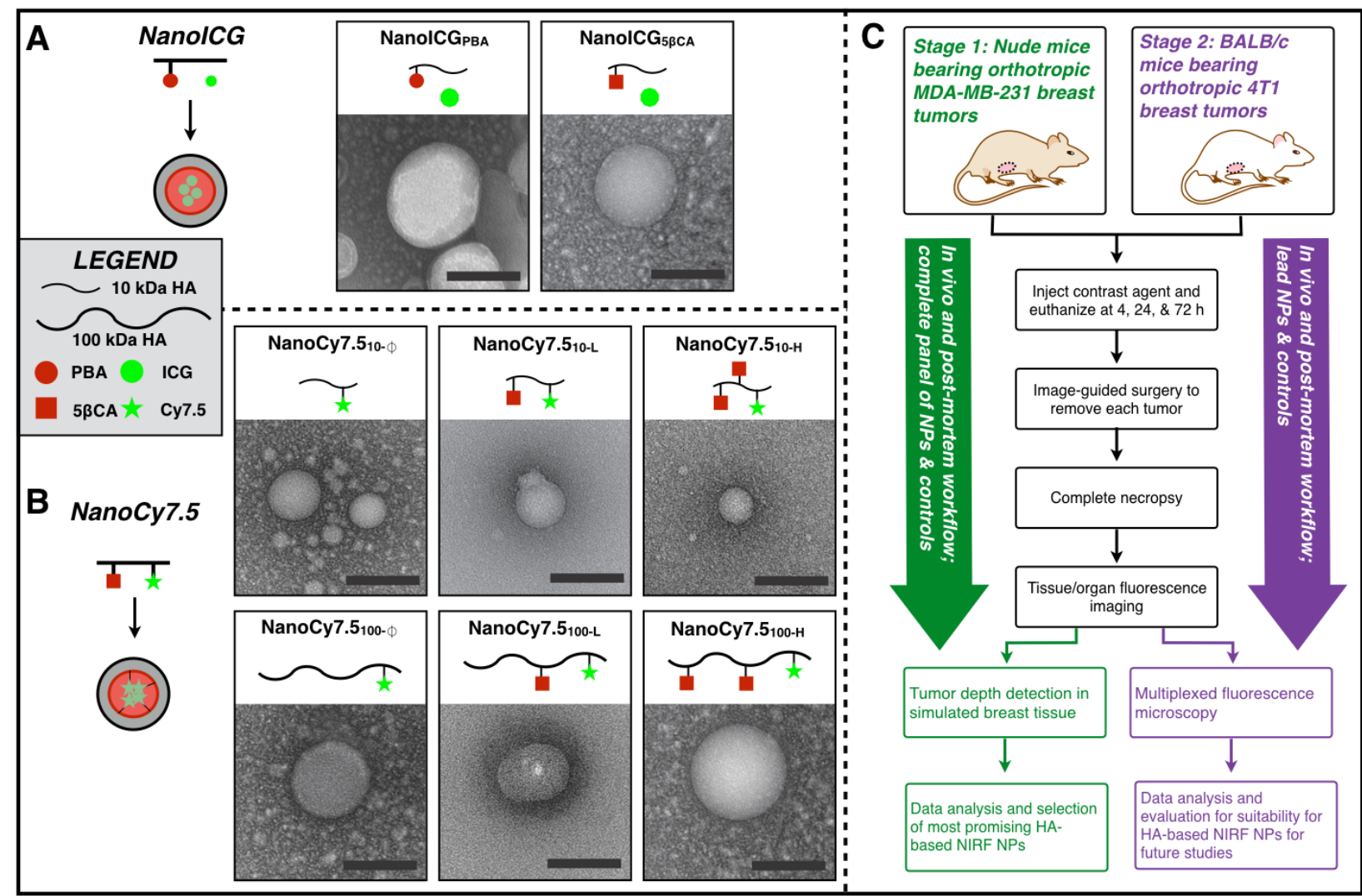

Figure 1. (A,B) Transmission electron microscopy images of NIRF HA-based NPs that were derived from $10 \mathrm{kDa}$ or $100 \mathrm{kDa}$ HA, using either PBA or $5 \beta C A$ as hydrophobic substituents to drive self-assembly (scale bars $=100 \mathrm{~nm}$ ). NanolCG formulations utilized physicochemically entrapped ICG, whereas NanoCy7.5 formulations utilized chemically conjugated Cy7.5. NP nomenclature is as follows: NanolCG refers to NP formulations of ICG. The subscript "5 $\beta$ CA" and "PBA" refer to the conjugated hydrophobic moieties, aminopropyl-5 $\beta$-cholanamide and aminopropyl-1-pyrenebutanamide, respectively. NanoCy7.5 refers to NP formulations of Cy7.5. The subscript "100" or "10" refers to 100 or 10 kDa HA, respectively. Subs "H", "L", and " $\varnothing$ " refer to high, low, or no 5BCA conjugation to HA. (C) These contrast agents were delivered to MDA-MB-231 tumor bearing mice, followed by extensive analysis of biodistribution, tumor contrast, relative contrast agent uptake, and tumor depth detection in tissue-mimicking phantoms. The most promising formulations were chosen based on these results for subsequent testing in immune-competent BALB/c mice bearing $4 T 1$ tumors, which were analyzed with multiplexed fluorescence microscopy in addition to similar analysis as the initial study. 


\section{Transmission Electron Microscopy}

NPs were dissolved in pure water to approximately $1 \mathrm{mg} / \mathrm{ml}$ and placed on Formvar/Silicone monoxide coated 200 mesh copper grids and allowed to adhere for approximately 2 minutes. Nanovan negative stain was applied for $30 \mathrm{~s}$ followed by blotting and imaging with a FEI Tecnai G2 Spirit TWIN operating at an accelerating voltage of $80 \mathrm{kV}$.

\section{Tumor Model Preparation}

All animal work was conducted within the guidelines of a protocol approved by the University of Nebraska Medical Center or the Wake Forest University Health Science Center IACUCs. MDA-MB-231 human breast cancer cells were grown in MEM with $10 \%$ FBS, 1X penicillin/streptomycin, L-glutamine, and $1 \mu \mathrm{g} / \mathrm{ml}$ puromycin, and express iRFP [26]. Cells were prepared for animal injection by mixing with 50:50 media:matrigel. Tumor xenografts were introduced to 11-13 week old female athymic nude mice (Jackson Labs; Bar Harbor, ME) by bilateral orthotopic injections into left and right inguinal mammary pads $\left(100 \mu \mathrm{L}\right.$ injections of $2 \times 10^{7}$ cells $\left./ \mathrm{ml}\right)$ and were allowed to grow at least three weeks; iRFP fluorescence could readily be detected from tumor xenografts as shown in Supplemental Figure 2. 4T1 tumors were grown in immune-competent BALB/c mice. 4T1 cells were prepared identically to MDA-MB-231 cells and injected into the left and right inguinal mammary pads of female BALB/c mice (Jackson Labs; Bar Harbor, ME), aged 11 weeks. Tumors were allowed to grow for 9-13 days prior to the mice being euthanized and imaged.

\section{In Vivo Near-infrared Contrast Agent Delivery and Imaging}

Contrast agents, including each NP formulation (containing $10 \mathrm{nmol}$ ICG or $1.2 \mathrm{nmol}$ Cy7.5) or corresponding dye at equivalent dose, were injected i.v. $(200 \mu \mathrm{l})$ via a tail vein. The quantity of ICG was equal across both NanoICG and ICG groups (10 nmol ICG/mouse), NanoCy7.5 and Cy7.5 groups were administered $1.2 \mathrm{nmol}$ Cy7.5/mouse due to the brightness of Cy7.5. Mice were then euthanized at the designated time point after injection; 4,24 , or 72 hours. After necropsy, mouse organ/tissue and tumor specimens were then imaged using a Pearl Trilogy Small Animal imaging system (LI-COR; Lincoln, NE) to detect ICG or Cy7.5 (800 nm channel) fluorescence from the contrast agents. Analysis was performed using LI-COR Image Studio 5.0 software to manually draw areas of interest around tumors and organs, and average pixel intensity was used to calculate signal to noise ratio (SNR) and contrast to noise ratio (CNR);
SNR $=$ (average tissue intensity per pixel in an area of interest)/(standard deviation of background area of interest). $\mathrm{CNR}=$ (Tumor SNR - Muscle SNR)/(standard deviation of background). Relative SNR of NP formulations groups to dye only groups was calculated as $\left(\mathrm{SNR}_{\mathrm{NP}}\right.$ group $) /\left(\mathrm{SNR}_{\text {dye }}\right.$ alone group), where the free dye is either ICG for comparison to NanoICG or Cy7.5 for comparison to NanoCy7.5. This metric was chosen to "normalize" the signal of each $\mathrm{NP}$ formulation to its respective free dye to determine whether or not NP formulation improves tumor delivery of dye and compare between NP formulations.

\section{Image-Guided Surgery}

BALB/c mice had orthotopic 4T1 tumors $\left(2 \times 10^{7}\right.$ cells in 50:50 media:matrigel) implanted bilaterally into thoracic mammary glands and allowed to grow as described above. NanoCy7.5100-H $(1.2 \mathrm{nmol} /$ mouse $)$ or NanoICG ${ }_{\text {PBA }}(10 \mathrm{nmol} /$ mouse) or their respective free dye controls at equivalent dye concentration were injected i.v. via a tail vein. Mice were then euthanized after $24 \mathrm{~h}$ and a simulated image-guided surgical procedure was performed. The bilateral mammary tumors were identified using a combination of visual resection and with the aid of a separate fluorescence image-guided surgery system, termed (FIGSS), resected under image-guidance, then followed by reexamination of the contrast enhancing region. FIGSS, which we have previously described in detail elsewhere [35,36], utilizes two separate, but integrated components. The first is a spectrophotometer that is fiber-coupled to a hand-held device that trasmits 785 $\mathrm{nm}$ excitation and collects wavelength-resolved fluorescence emission through the same device [35]. The version used here is capable of 8 (low), 30 (medium low), 80 (medium), 150 (medium high), and 200 (high) $\mathrm{mW}$ power and collects spectra between $800-950 \mathrm{~nm}$. The second component of the system is a real-time video widefield imaging system that collects both NIR emission from the contrast agent and visibile light for anatomical reference [36]. The widefield sytem uses the laser from the spectroscopic system as its excitation source. NanoCy7.5 and Cy7.5 required low power $(8 \mathrm{~mW})$ excitation, while NanoICG and ICG required medium power $(80 \mathrm{mw})$ excitation. Harvested tissue was subjected to additional spectroscopy and imaging analysis. Spectra from tissues were obtained with the tip of the laser $1 \mathrm{~cm}$ from the tissue surface.

\section{Construction of Breast Tissue Mimicking Phantoms}

Breast tissue mimicking phantoms were constructed as described elsewhere $[37,38]$. Briefly, 
$10 \%$ gelatin powder (Spectrum; New Brunswick, NJ), was dissolved in $50 \mathrm{mM}$ Tris- $\mathrm{HCl}$ with $15 \mathrm{mM} \mathrm{NaN}_{3}$. This was dissolved by stirring at $50{ }^{\circ} \mathrm{C}$, followed by cooling to $33-37^{\circ} \mathrm{C}$. Dried bovine erythrocytes (Sigma; St. Louis, MO) were then added to achieve $17 \mu \mathrm{M}$ hemoglobin, followed by $1 \%$ (v/v) Intralipid 20\% (Sigma; St. Louis, MO). This mixture was then aliquoted into tissue culture dishes to achieve thicknesses of $2,3,4,5$, or $6 \mathrm{~mm}$, and placed at $4{ }^{\circ} \mathrm{C}$ until ready for use. Tumor fluorescent signal penetration through tissue phantoms was analyzed using FIGSS. Initial readings with no phantoms were taken. Phantoms which measured between 2-6 mm thick in $1 \mathrm{~mm}$ increments were then placed sequentially over the tumors and imaged using 30 $\mathrm{mW}$ laser excitation. Images taken using the IGS system were rated with a qualitative "detection factor" (DF) using a strong $(+++)$, moderate $(++)$, weak $(+)$, and absent (-) scale. Color and NIR channel images were saved separately followed by processing with ImageJ (NIH; Bethesda, MD). All images were processed identically.

\section{Histological Analysis}

\section{NIR fluorescent and H\&E samples from frozen sections}

Tumor tissue from image-guided surgery studies was immediately placed in OCT mounting media gel and rapidly frozen in liquid nitrogen. These samples were then cut and processed by the UNMC Tissue Sciences Facility. Sections from each sample were stained with hematoxylin and eosin (H\&E) or were left unstained for NIR fluorescence microscpy. Samples were analyzed with an Olympus IX73 Inverted Microscope with a xenon excitation source and imaged with an Olympus DP80 Digital Camera and cellSens Dimension software. H\&E-stained tissue samples were visualized and imaged with brightfield microscopy. Unstained tissue samples were visualized and imaged for autofluorescence (FITC filter cube) and NIR fluorescence (ICG filter cube). Exposure times of each channel were manually adjusted to maintain clear image resolution. Background signal from images in the autofluorescence and NIR channels were black-balanced on an area that contain tissue using an automated algorithm provided by cellSens Dimension software. NIR signal was pseudo-colored yellow, but with no additional signal processing, using ImageJ software (https://imagej.nih.gov/ij/).

\section{Multiplexed analysis on formalin fixed paraffin embedded sections}

Tissues from the biodistribution study using 4T1 tumors in BALB/c mice were excised and placed in
$10 \%$ neutral buffered formalin (Fisher Scientific; Waltham, MA) for 24-48 h, followed by placement in $70 \% \mathrm{EtOH}$ until processing. Paraffin embedded tissues were sectioned at $4 \mu \mathrm{m}$, baked at $57^{\circ} \mathrm{C}$ for 30 minutes, deparaffinized, permeabilized with $0.2 \%$ Triton X-100 (Sigma) and put through microwave antigen retrieval using AR6 buffer (Perkin Elmer; Waltham, MA). Tumor sections and control tissue were blocked for 10 minutes with serum free protein block (Dako; Carpenteria, CA), and then stained in antibody diluent (Dako) overnight at $4^{\circ} \mathrm{C}$ with primary antibody. Following several washes with Tris buffered saline-TWEEN (TBST), sections were then stained with secondary (HRP) antibody (Perkin Elmer) for 1 hour at room temperature. Following several washes with TBST, sections were then treated with TSA Plus working solution and selected fluorophore (Perkin Elmer) for $10 \mathrm{~min}$ at room temperature. Following several washes, sections are put back into microwave antigen retrieval. Repeating blocking, primary antibody incubation, HRP incubation and TSA Plus reaction for all remaining antibodies. Antibodies used included alpha smooth muscle actin (stroma marker) and pan cytokeratin (tumor marker) in 1:50 dilutions (Abcam; Cambridge, UK). Slides were then washed several times and stained with DAPI (Perkin Elmer) for 5 minutes. Slides were then washed with TBST. Prolong gold mounting medium (Life Technologies; Carlsbad, CA) was used for coverslip application. Tissue samples were segmented using InForm software (Perkin Elmer). The software is able to be trained to identify patterns in the tissue and creates an algorithm to identify these patterns. Software is trained to distinguish between tumor, stroma, and blank areas.

\section{Statistical Analysis}

Data was analyzed in Prism 6 software (Graphpad; La Jolla, CA). Data from the LI-COR Pearl system was converted to Excel for processing. Errors were propagated according to established rules from averaged SNR values. Biodistribution was analyzed using two-way ANOVA with multiple comparisons and Tukey's test. MDA-MB-231 tumor CNR and $\mathrm{SNR}_{\mathrm{NP}} / \mathrm{SNR}_{\text {dye }}$ ratios were analyzed using one-way ANOVA with multiple comparisons with Tukey's test. For BALB/C mice bearing 4T1 tumors biodistribution was analyzed using one-way ANOVA and $C N R$ and $S_{N R} / S_{N P R}$ dye ratios were analyzed using Student's t-test. Significant differences were considered as $p<0.01$. Measurements of individual tumors were used for determination of tumor sample size (range: $N=4-10$ depending on tumor growth). P-values presented for ANOVA tests are multiplicity adjusted p-values. All graphs show groups with Mean 
\pm SD. Significance levels are represented as follows: **, $\mathrm{p}<0.01 ;{ }^{* * *}, \mathrm{p}<0.001 ;{ }^{* * * *} \mathrm{p}<0.0001$.

\section{Results}

\section{Relative Biodistribution}

The relative biodistribution, as calculated by SNR values of all HA-based NIRF NP formulations and free dye, are shown in Figure 2A-I, and exact values and significance levels are shown in Supplemental Table 1. Overall fluorescence signal, including that emitting from the MDA-MB-231 tumors, was higher with Cy7.5-based agents compared to ICG-based NPs (Figure 2J). The highest SNRs were observed in the liver and kidneys for ICG and NanoICG formulations, which reduced rapidly between 4 and $24 \mathrm{~h}$. Initially, ICG alone group has

\section{$4 \mathrm{~h}$}
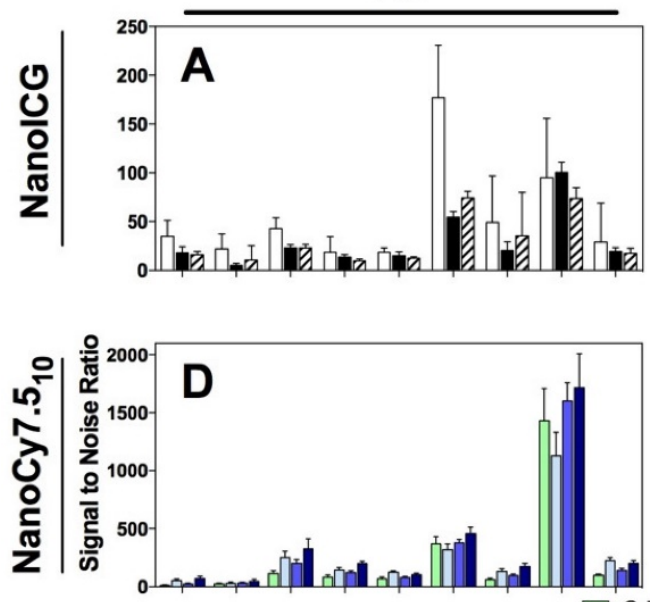

$\square$ Cy7.5 $\square$ NanoCy7.5 $10-\varnothing \quad \square$ NanoCy7.5 $10-\downarrow$

$24 \mathrm{~h}$ much higher signal in the kidney than NanoICG. NanoICG signal decreases in the kidney, but more slowly after the $4 \mathrm{~h}$ time point. The higher signal in kidney, liver, and spleen of the NP formulations of dye at later time points likely suggests that the NP formulation impacts biodistribution. The NIR signal in the kidney for most NanoCy7.5 agents was similar or lower than free Cy7.5 group indicating that HA governed Cy7.5 biodistribution, which is consistent with the stability study in Supplementary Figure 1. Higher signal in the lung at $4 \mathrm{~h}$ was also observed among NanoCy7.5 formulations, which dramatically decreased by 24 h. 100 kDa NanoCy7.5 formulations were unique in showing very high signal in the spleen at both 4 and $24 \mathrm{~h}$. The relative signal in the bone was also observed to be higher with $100 \mathrm{kDa}$ Cy7.5
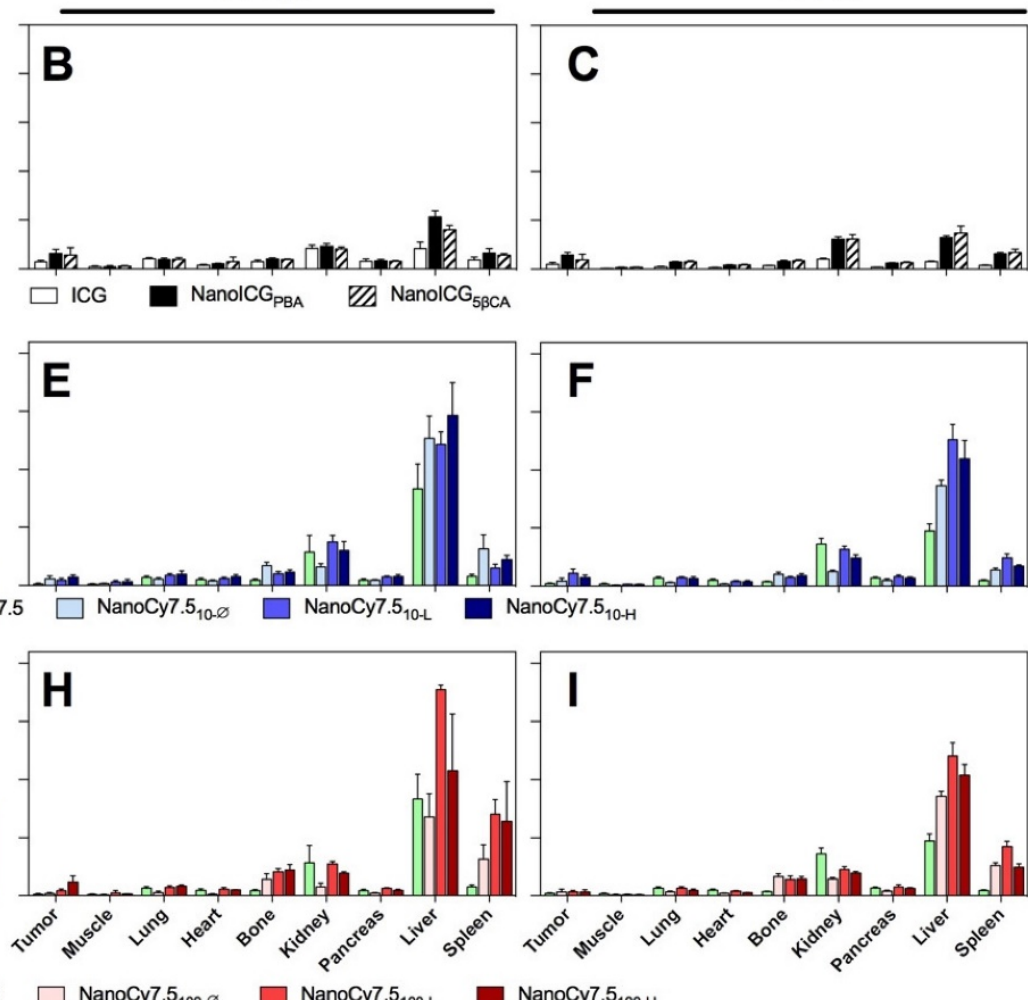

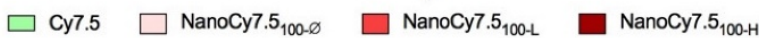

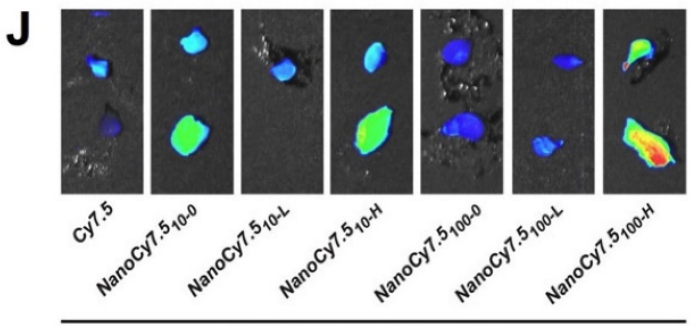

Cy7.5-Based Agents (1.2 nmol Cy7.5/mouse)

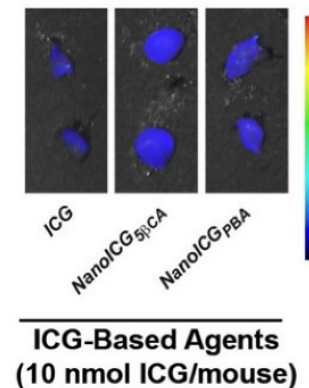

Figure 2. Biodistribution of contrast agents at 4,24 , and $72 \mathrm{~h}$ after administration in MDA-MB-231 tumor bearing nude mice. $\mathrm{N}=4-10$ tumors, which depended on tumor growth in mice. Bars represent average pixel SNR (mean $\pm S D$ ). For clarity, significant differences are described in the supplementary information. (A-C) NanolCG, (D-F) NanoCy7.5 based on $10 \mathrm{kDa}$ HA, and (G-I) NanoCy7.5 based on $100 \mathrm{kDa}$ HA at $4 \mathrm{~h}$ (left), $24 \mathrm{~h}$ (middle), and $72 \mathrm{~h}$ (right) after contrast agent injection. (J) NIR image of tumors harvested from mice. All tumors in J are presented with the same colormap to visualize differences between groups. Supplemental Figure 2 shows representative NIR images of all harvested organs. 
formulations than for other groups. It should be noted that ICG present in NanoICG is physically entrapped, and thus the fluorescence signal from organs with either NanoICG formulations may not represent localization or clearance of associated HA polymers, whereas Cy7.5 is covalently attached to HA in NanoCy7.5 formulations. Supplementary Figure 1 shows Cy7.5 is slowly released from NanoCy7.5100-H, while ICG is rapidly released into serum, indicating the that the NPs formulation of ICG has a less significant effect than when a dye is directly conjugated to amphiphilic HA.

\section{Tumor Contrast}

No significant differences were found between ICG-containing groups at $4 \mathrm{~h}$, shown in Figure 3A. ICG CNR was lower than NanoICGPBA at $24 \mathrm{~h}(\mathrm{p}<$ $0.01)$ and $72 \mathrm{~h}(\mathrm{p}<0.0001)$ (Figure 3B\&C). NanoCy7.510-ø and NanoCy7.510-H had higher CNR than Cy7.5 at $4 \mathrm{~h}(\mathrm{p}<0.001)$. NanoCy7.510-L had a lower CNR than other NanoCy7.5 (10kDa) formulations at $4 \mathrm{~h}(\mathrm{p}<0.01)$. Both Cy7.5 and NanoCy7.510-L showed negative contrast at $4 \mathrm{~h}$, indicating that, on average, muscle tissue had greater signal than tumor. No significant difference was observed at $24 \mathrm{~h}$ among $10 \mathrm{kDa}$ Cy7.5 conjugates at 24 $\mathrm{h}$. The highest contrast $(93.5 \pm 37.1)$ was observed in Cy7.510-L at $72 \mathrm{~h}$, which was greater than Cy7.5 (p < $0.0001)$ and NanoCy7.5 $10-\varnothing(\mathrm{p}<0.01)$. NanoCy7.5100-L showed higher signal than both Cy7.5-amine and Cy7.5100-H at $4 \mathrm{~h}$. At $24 \mathrm{~h}$, NanoCy7.5100-H had higher CNR than all other groups ( $p<0.0001$ vs. Cy7.5), $(p<$ 0.001 vs. NanoCy7.5100-ø), ( $\mathrm{p}<0.01$ vs. NanoCy7.5100-L). However, by $72 \mathrm{~h}$ tumor contrast between the three NanoCy7.5100 conjugates was not significant.

The CNR of each NP formulations was higher compared to their respective free dyes at 24 and $72 \mathrm{~h}$. High variability in tumor and muscle signal was observed among all groups at $4 \mathrm{~h}$, resulting in CNR values with large SD; several individual CNR values were negative indicative of a higher signal in muscle than tumor. The CNR for both NanoICG formulations was consistent between 24 and $72 \mathrm{~h}$. NanoCy7.510-L showed higher CNR at $72 \mathrm{~h}$ and NanoCy7.5100-H showed higher $\mathrm{CNR}$ at $24 \mathrm{~h}$. The magnitude of contrast was higher in NanoCy7.5 conjugates compared to NanoICG, despite using approximately one-tenth of the dose.
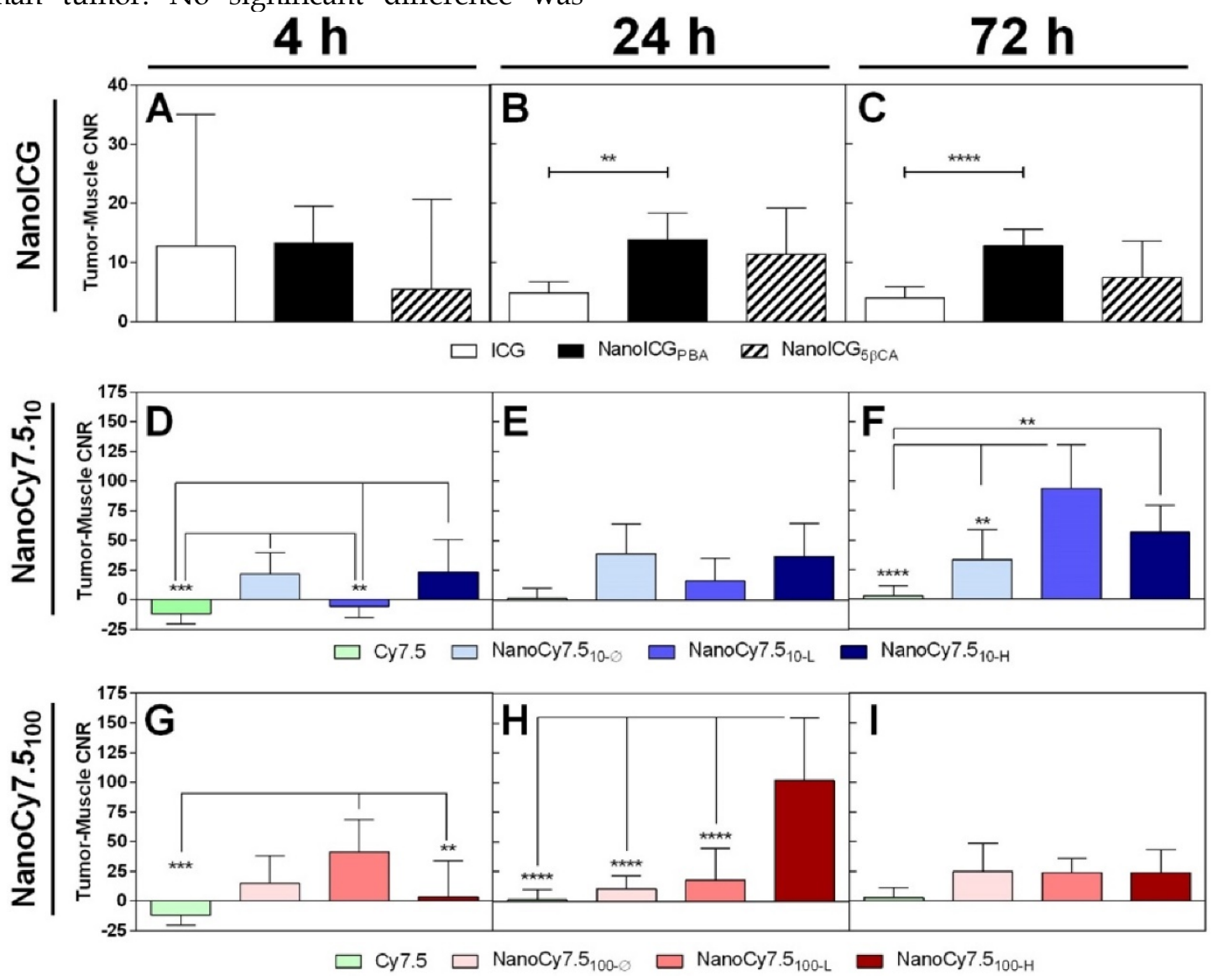

Figure 3. Contrast between tumor and muscle tissue of MDA-MB-231 tumors grown in nude mice. CNR was variable at $4 \mathrm{~h}$, indicating a wide range of individual tumor and muscle signal levels. Levels stabilized by $24 \mathrm{~h}$ with CNR > 10 for NanolCGPBA and > 100 for NanoCy7.5 100-H at $24 \mathrm{~h}$ (highest observed). CNR was reduced in NanolCG and NanoCy7.5 ${ }_{100}$ formulations by $72 \mathrm{~h}$, however NanoCy7.5/0 formulations showed higher CNR values, with NanoCy7.5/0-L being greatest at 72 h. Data was analyzed using one-way ANOVA with multiple comparisons and Tukey's post-test (mean $\pm \mathrm{SD}$ ). 


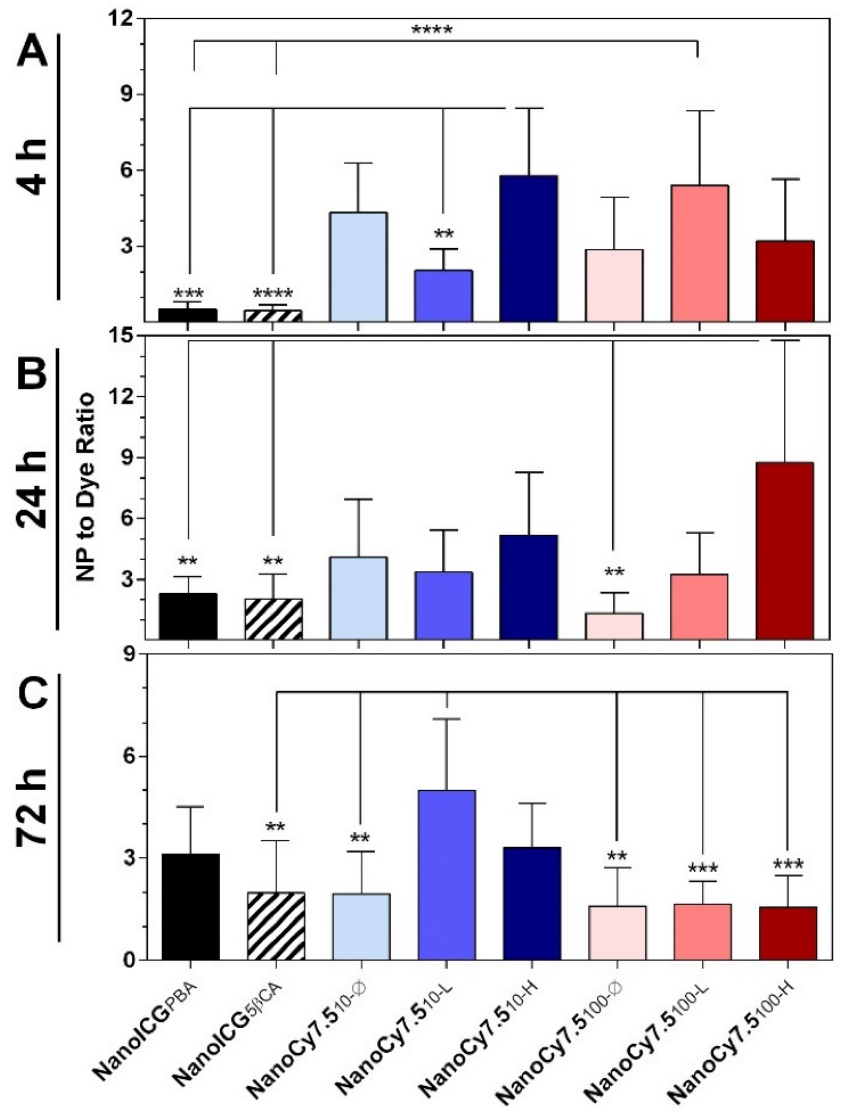

Figure 4. $S N R_{N P} / S N R_{\text {dye }}$ ratio shows the effect of tumor delivery due to the NP formulation. With the exception of NanolCG formulations at $4 \mathrm{~h}$, all NP formulations showed $\mathrm{SNR}_{N P} / \mathrm{SNR}_{\text {dye }}>1$, indicating greater contrast agent accumulation in tumors due to the NP formulation itself. Greatest results were observed with NanoCy7.5100- $\mathrm{H}$ at $24 \mathrm{~h}$, and NanoCy7.5 $10-\mathrm{H}$ at $4 \mathrm{~h}$. Data was analyzed using one-way ANOVA using multiple comparisons and Tukey's post-test (mean \pm SD).

\section{Effect of NP Formulation}

In order to compare the relative differences in imaging tumors between NP formulations and free dyes, we examined the ratio of the NP SNR to the SNR of their respective free dye (ICG for NanoICG, and Cy7.5-amine for NanoCy7.5 formulations), identified as $\mathrm{SNR}_{\mathrm{NP}} / \mathrm{SNR}_{\text {dye. }}$. For example, a value of $\mathrm{SNR}_{\mathrm{NP}} / \mathrm{SNR}_{\text {dye }}=0.5$ indicates that the NP formulation provides one-half the tumor per-pixel SNR compared to free dye, while a value of $\mathrm{SNR}_{\mathrm{NP}} / \mathrm{SNR}_{\text {dye }}=2$ indicates the NP per-pixel SNR was twice as large as the signal from the free dye group. At $4 \mathrm{~h}$, the two formulations with the greatest $\mathrm{SNR}_{\mathrm{NP}} / \mathrm{SNR}_{\text {dye }}$ ratio were NanoCy7.510-H $(5.8 \pm 0.8)$ and NanoCy7.5 $100-\mathrm{L}$ (5.4 \pm 2.9 ) (Figure 4A). At $4 \mathrm{~h}$, NanoICG formulations had values below 1 , indicating free ICG had higher SNR. By $24 \mathrm{~h}$ NanoCy7.5100-H showed the highest ratio at 8.7 \pm 6.0 times greater than its respective free dye, Cy7.5 (Figure 4B). This was the greatest observed among all contrast agents at any time point tested. All formulations showed $\mathrm{SNR}_{\mathrm{NP}} / \mathrm{SNR}_{\text {dye }}>1$ beyond $24 \mathrm{~h}$. At $72 \mathrm{~h}$, the largest ratio was NanoCy7.510-L $(5.0 \pm 2.1)$, which was significantly larger than any $100 \mathrm{kDa}$ formulation, as well as NanoICG $5 \beta C A$ and NanoCy7.510-ø (Figure 4C). With the exception of the NanoICG formulations at $4 \mathrm{~h}$, all formulations showed $\mathrm{SNR}_{\mathrm{NP}} / \mathrm{SNR}_{\text {dye }}>1$, indicating that the NP formulations had higher SNR within tumors compared to their respective free dyes.

\section{Tumor Detection in Breast Tissue Mimicking Phantoms}

An IGS system was used to examine the tumor signals and estimate the maximum depth at which a tumor could be detected. DF, a qualitative measure, was used to determine the visual level of detectability of tumors lying beneath breast phantoms of varying thicknesses. Figure 5 shows a representative tumor providing signal through increasingly thick phantoms ranging from $0 \mathrm{~mm}(\mathrm{~A}), 2 \mathrm{~mm}(\mathrm{~B}), 3 \mathrm{~mm}(\mathrm{C})$, and 4 $\mathrm{mm}(\mathrm{D})$. The corresponding fluorescence spectra and DF are shown adjacent. DFs of strong $(+++)$, moderate $(++)$, weak $(+)$, and absent (-) for all contrast agents at all phantom thicknesses are shown in Table 1. As expected, signal strength decreased with increasing phantom thickness among all contrast agents. Within groups of a single formulation, earlier time points tended to show greater detectability at depth compared to later time points. Regardless of time point, most formulations were not detectable beyond $4 \mathrm{~mm}$ deep, with only a single tumor treated with NanoCy7.5100-L at $4 \mathrm{~h}$ being detectable through a $6 \mathrm{~mm}$ phantom. Free dyes (ICG and Cy7.5) and NanoICG formulations were less detectable than NanoCy7.5 formulations. Most NanoCy7.5 groups were detectable to $3 \mathrm{~mm}$ or more, and often up to $4 \mathrm{~mm}$. There was also notable heterogeneity in detectability between individual tumors of each group.

\section{Lead Formulation Evaluation in Immune Competent Mice}

One ICG-based and one Cy7.5-based NP formulation was identified for further evaluation based on the studies with mice bearing MDA-MB-231 tumors. NanoCy7.5100-H was chosen as the lead Cy7.5-conjugated formulation due to the large CNR at $24 \mathrm{~h}$. NanoICG $\mathrm{CNR}$ and to continue development of improved formulations of a clinically-approved NIR dye. SNR-based biodistribution studies in BALB/c mice bearing 4T1 tumors showed similar results to that observed previously, i.e. higher SNR observed with Cy7.5 and NanoCy7.5 $100-\mathrm{H}$ compared to ICG and NanoICGPBA (Figure 6). Between organs, higher signals were seen in liver, kidney, and spleen, which decreased between 4 and $24 \mathrm{~h}$. Exact values and significance levels are found in Supplementary Table 2. Notably, NanoICG had greater tumor SNR than 
ICG alone at $24 \mathrm{~h}(\mathrm{p}<0.001)$, and NanoCY7.5100-H had greater tumor SNR than Cy7.5 alone at both 4 and $24 \mathrm{~h}$ $(\mathrm{p}<0.0001)$.

Table 1. Detection of excised MDA-MB-231 tumors through simulated breast tissue phantoms. ${ }^{a}$

\begin{tabular}{|c|c|c|c|c|c|c|c|}
\hline \multirow[t]{2}{*}{ Contrast agent } & \multirow[t]{2}{*}{ Time (h) } & \multicolumn{6}{|c|}{ Phantom Thickness (mm) } \\
\hline & & 0 & 2 & 3 & 4 & 5 & 6 \\
\hline \multirow[t]{3}{*}{ ICG } & 4 & +++ & ++ & + & - & - & - \\
\hline & 24 & + & - & - & - & - & - \\
\hline & 72 & ++ & + & - & - & - & - \\
\hline \multirow[t]{3}{*}{ NanoICG } & 4 & ++ & + & - & - & - & - \\
\hline & 24 & ++ & + & - & - & - & - \\
\hline & 72 & +++ & ++ & - & - & - & - \\
\hline \multirow[t]{3}{*}{ NanoICG $5 \beta C A$} & 4 & ++ & + & - & - & - & - \\
\hline & 24 & ++ & + & - & - & - & - \\
\hline & 72 & ++ & + & - & - & - & - \\
\hline \multirow[t]{3}{*}{ Cy7.5 } & 4 & + & - & - & - & - & - \\
\hline & 24 & ++ & + & - & - & - & - \\
\hline & 72 & ++ & + & - & - & - & - \\
\hline \multirow[t]{3}{*}{ NanoCy7.510-ø } & 4 & +++ & +++ & ++ & + & - & - \\
\hline & 24 & +++ & +++ & ++ & + & - & - \\
\hline & 72 & +++ & ++ & + & - & - & - \\
\hline \multirow[t]{3}{*}{ NanoCy7.510-L } & 4 & +++ & +++ & ++ & + & - & - \\
\hline & 24 & +++ & +++ & ++ & + & - & - \\
\hline & 72 & +++ & +++ & ++ & + & - & - \\
\hline \multirow[t]{3}{*}{ NanoCy7.510-H } & 4 & +++ & +++ & ++ & + & - & - \\
\hline & 24 & +++ & ++ & + & - & - & - \\
\hline & 72 & +++ & ++ & + & - & - & - \\
\hline \multirow[t]{3}{*}{ NanoCy7.5100-Ø } & 4 & +++ & ++ & + & - & - & - \\
\hline & 24 & +++ & + & - & - & - & - \\
\hline & 72 & +++ & ++ & + & - & - & - \\
\hline \multirow[t]{3}{*}{ NanoCy7.5100-L } & 4 & +++ & +++ & +++ & +++ & ++ & + \\
\hline & 24 & +++ & ++ & ++ & + & - & - \\
\hline & 72 & +++ & ++ & + & - & - & - \\
\hline \multirow[t]{3}{*}{ NanoCy7.5100-H } & 4 & +++ & +++ & ++ & + & - & - \\
\hline & 24 & +++ & +++ & ++ & + & - & - \\
\hline & 72 & +++ & ++ & + & - & - & - \\
\hline
\end{tabular}

aLaser power set to $30 \mathrm{~mW}$; distance between phantom material and laser tip was 1 $\mathrm{cm}$

Tumor-to-muscle CNR also showed similar results to those seen in nude mice, with the highest contrast observed with NanoCy7.5100-H at both 4 and $24 \mathrm{~h}$, with CNR values of $187 \pm 58(4 \mathrm{~h})$ and $246 \pm 79$ $(24 \mathrm{~h})$ (Figure 7). These levels were significantly and meaningfully greater than those observed using ICG or NanoICG ${ }_{P B A}$, which ranged from $12 \pm 3$ to $48 \pm 12$. NanoICG had significantly greater CNR than ICG at $24 \mathrm{~h}(12 \pm 3$ vs $27 \pm 3, \mathrm{p}<0.0001)$. A $72 \mathrm{~h}$ time point was not included with the lead contrast agents because overall signal was lower in the MDA-MB-231 xenografts compared to $24 \mathrm{~h}$. Furthermore, $4 \mathrm{~h}$ and 24 $\mathrm{h}$ more practical for further preclinical and potential clinical development of image-guided surgery contrast agents.

The ratio of $\mathrm{SNR}_{\mathrm{NP}} / \mathrm{SNR}_{\text {dye }}$ of NanoICGPBA was $0.9 \pm 0.3$ at $4 \mathrm{~h}$, and $2.2 \pm .0 .5$ at $24 \mathrm{~h}$, indicating similar SNR for NanoICGPBA and ICG at $4 \mathrm{~h}$, and approximately double that of free ICG at $24 \mathrm{~h}$, respectively (Figure 8). NanoCy7.5100-H showed significantly larger $\mathrm{SNR}_{\mathrm{NP}}$ to $\mathrm{SNR}_{\text {dye }}$ ratios at both 4 and $24 \mathrm{~h}$ compared to NanoICGPBA. NanoCy7.5100-H SNR was $4.8 \pm 1.6$ times greater than Cy7.5 at $4 \mathrm{~h}$, and $9.3 \pm 2.9$ times greater than Cy7.5 at $24 \mathrm{~h}$. This indicates that not only did NanoCy7.5100-H have greater contrast than either NanoICGPBA or Cy7.5, but also that it resulted in higher relative tumor signal compared to groups with dye only injection.

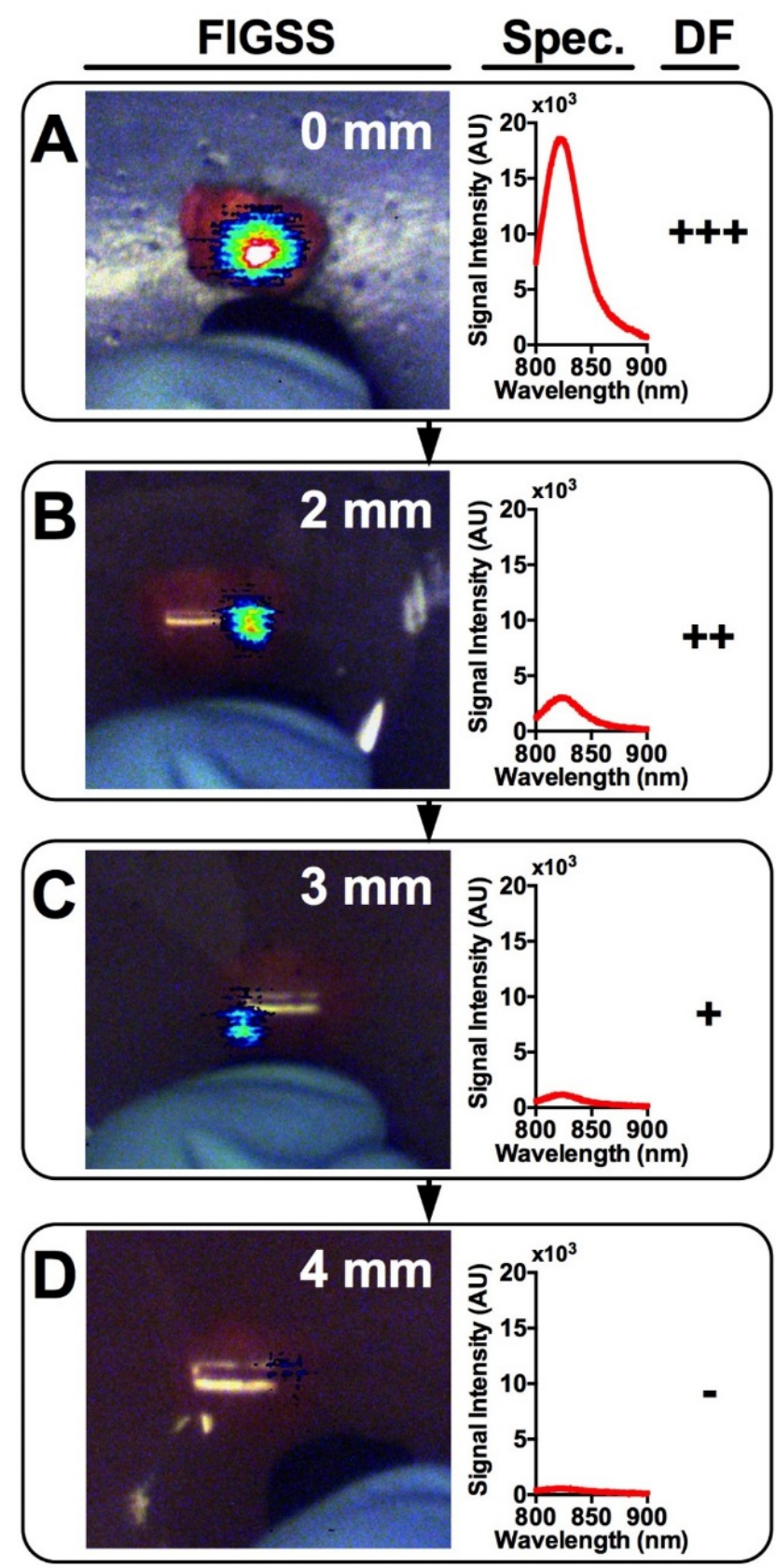

Figure 5. Representative images and spectra showing IGS-system imaging of a single tumor with underlying increasingly thick tissue mimicking phantoms. Signal is strongest when the tumor is completely exposed $(A)$ and rapidly diminishes with increasing phantom thickness (B-D). Detection Factor (DF) represents the qualitative measure that surgeons would have available and could be used to investigate suspect regions in the operating field. 


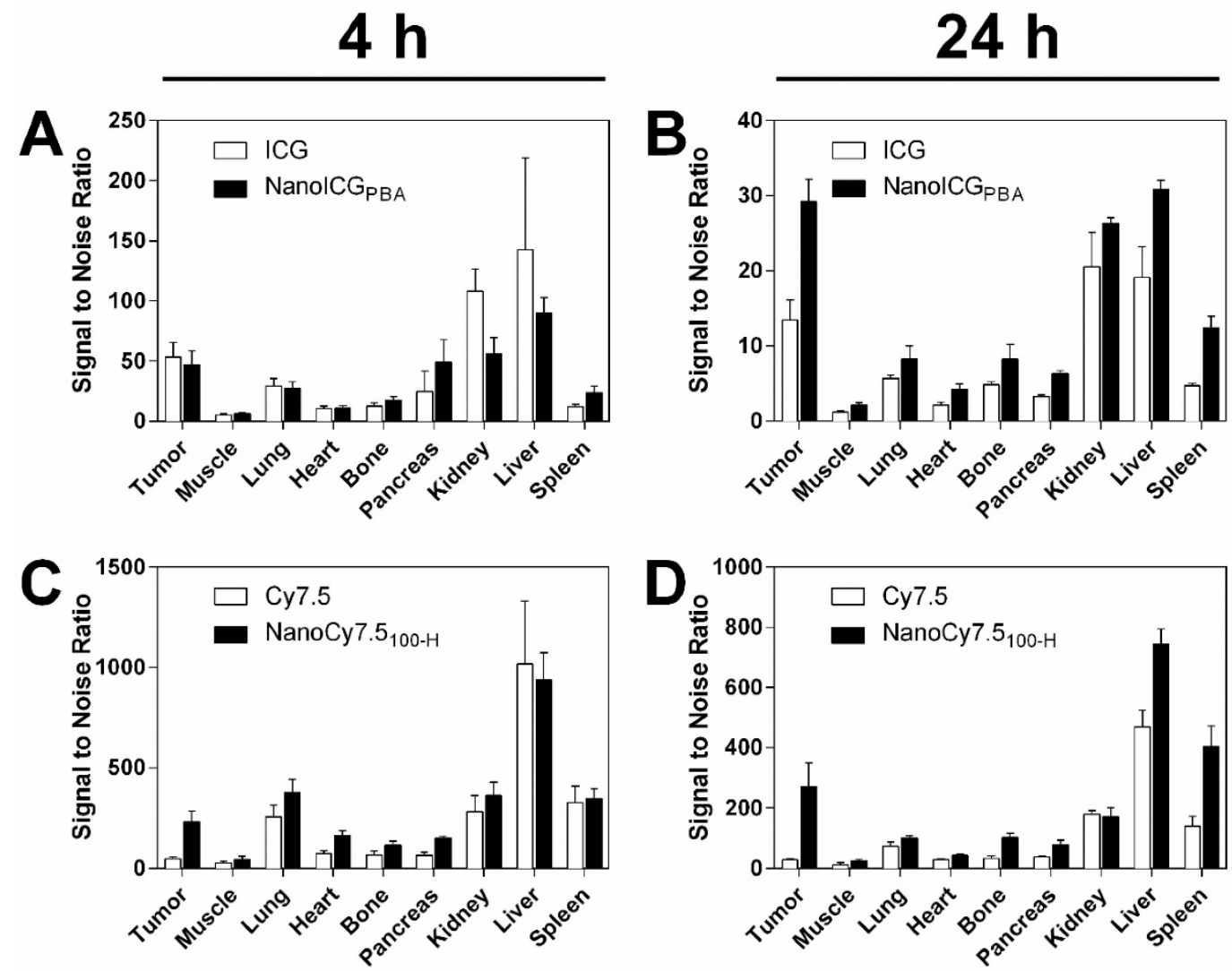

Figure 6. Biodistribution of contrast agents in 4T1 tumors showed similar results compared to MDA-MB-231 tumors. SNR of both ICG and NanolCG was higher in all tissues at $4 \mathrm{~h}(\mathrm{~A})$, however signal remained higher in the tumor at $24 \mathrm{~h}$ with NanolCGpBA compared to ICG alone (B). Similar results were observed between NanoCy7.5 $100-\mathrm{H}$ and Cy.7.5 alone between $4 \mathrm{~h}(C)$, and $24 \mathrm{~h}(\mathrm{D})$. These results suggest that the biodistribution is not meaningfully different in immune competent mice. NanolCGPBA had greater tumor SNR than ICG alone at $24 \mathrm{~h}(\mathrm{p}<0.001)$, and NanoCy7.5100-H had greater tumor SNR than Cy7.5 alone at both 4 and $24 \mathrm{~h}$ ( $\mathrm{p}<0.0001)$. Data was analyzed using Student's t-test.
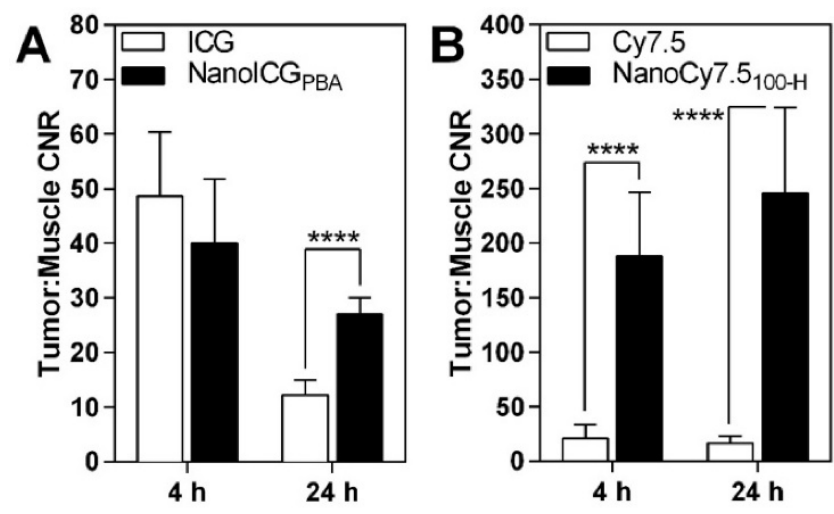

Time Post-injection

Figure 7. CNR levels were positive for all contrast agents, indicating greater signal in the tumor than muscle. CNR of ICG and NanoICG were not significantly different at $4 \mathrm{~h}$, however by $24 \mathrm{~h}$ NanolCG showed significantly greater contrast $(\mathrm{p}<0.0001)$. NanoCy7.5 showed significantly greater contrast than Cy7.5-amine at both 4 and 24 h $(p<0.0001)$. Data was analyzed using Student's t-test (mean $\pm S D)$.

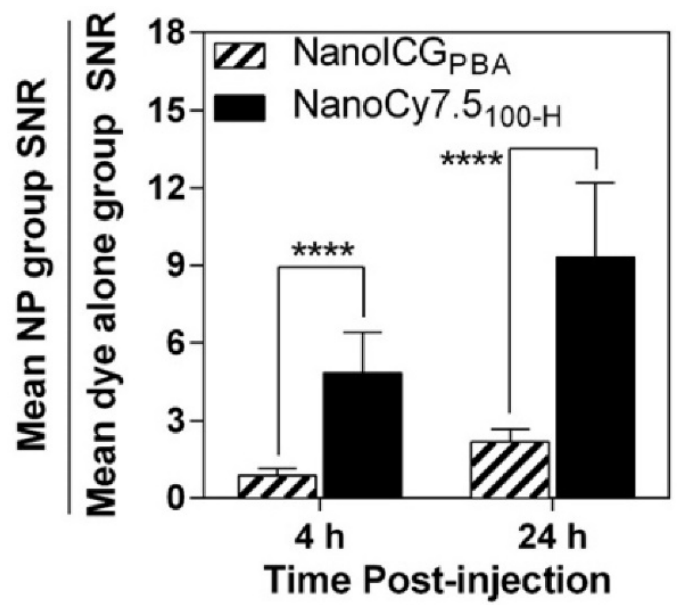

Figure 8. SNR $\mathrm{NP}_{\mathrm{SN}} / \mathrm{SR}_{\text {dye }}$ for both NanolCGPBA and NanoCy7.5 $100-\mathrm{H}$ at 4 and $24 \mathrm{~h}$ in 4T1 tumors was similar to that seen in MDA-MB-231 tumors, indicating a similar relative distribution of NP to free dye for both models. NanoCy7.5 $100-\mathrm{H}$ showed a higher SNR ${ }_{N P} / S N R_{\text {dye }}$ ratio for both 4 and $24 \mathrm{~h}$ than NanolCG $(\mathrm{p}<0.0001)$, indicating that this formulation showed greater accumulation in the tumor. Data was analyzed using Student's t-test (mean \pm SD). 


\section{Image-Guided Surgery}

The two lead NIR HA-NP formulations, NanoCy7.5100-H and NanoICG ${ }_{\text {рва }}$ and their respective dye-only controls were further examined for suitability for image-guided surgery. Intraoperative imaging provided by FIGSS showed that NIR signal could be detected in orthotopic 4T1 tumors during surgery (Figure 9A), while areas off the tumor did not enhance when the excitation laser was not directed at those regions (Figure 9A inset). Keeping the wide-field imaging parameters constant, NanoCy7.5 ${ }_{100-\mathrm{H}}(1.2 \mathrm{nmol} / \mathrm{mouse})$ was readily detected in tumor with $8 \mathrm{~mW}$ "low" excitation power, while NanoICG ${ }_{\text {pвA }}(10 \mathrm{nmol} /$ mouse) required $80 \mathrm{~mW}$ "medium" excitation power for similar intraoperative detection. Cy7.5 and ICG controls at the same dose, excitation power, and wide-field imaging parameters, resulted in no (for Cy7.5) and minimal (for ICG) detection of tumor during surgery. Figure 9B shows the intraoperative (ex vivo) analysis by FIGSS of resected tissue, confirmed to be tumor (Figure 9C), that enhanced in situ remained fluorescent during $e x$ vivo analysis demonstrating that both NanoCy7.5 and NanoICG could be used to detect tumor during surgery and provide further information in the operating room. Spectroscopic analysis provided by FIGSS showed that NanoCy7.5 resulted in a 13.9-fold stronger signal, based on AUC of the intensity vs. wavelength curve, compared to Cy7.5 control and 21.3-fold stronger signal compared to NanoICG. For each NP, spectroscopic measurements obtained from biopsies of non-enhancing regions during surgery, later determined to be muscle by histology, had low spectroscopic signal. Furthermore, NIRF microscopy of frozen section samples showed signal for

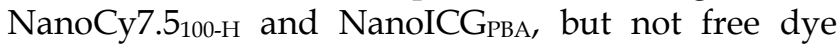
controls (Figure 9D-G). Taken together, wide-field imaging can be adjusted to optimize contrast enhancement for a given contrast agent, while wavelength-resolved NIR measurements by spectroscopic pen can provide semi-quantitative analysis.

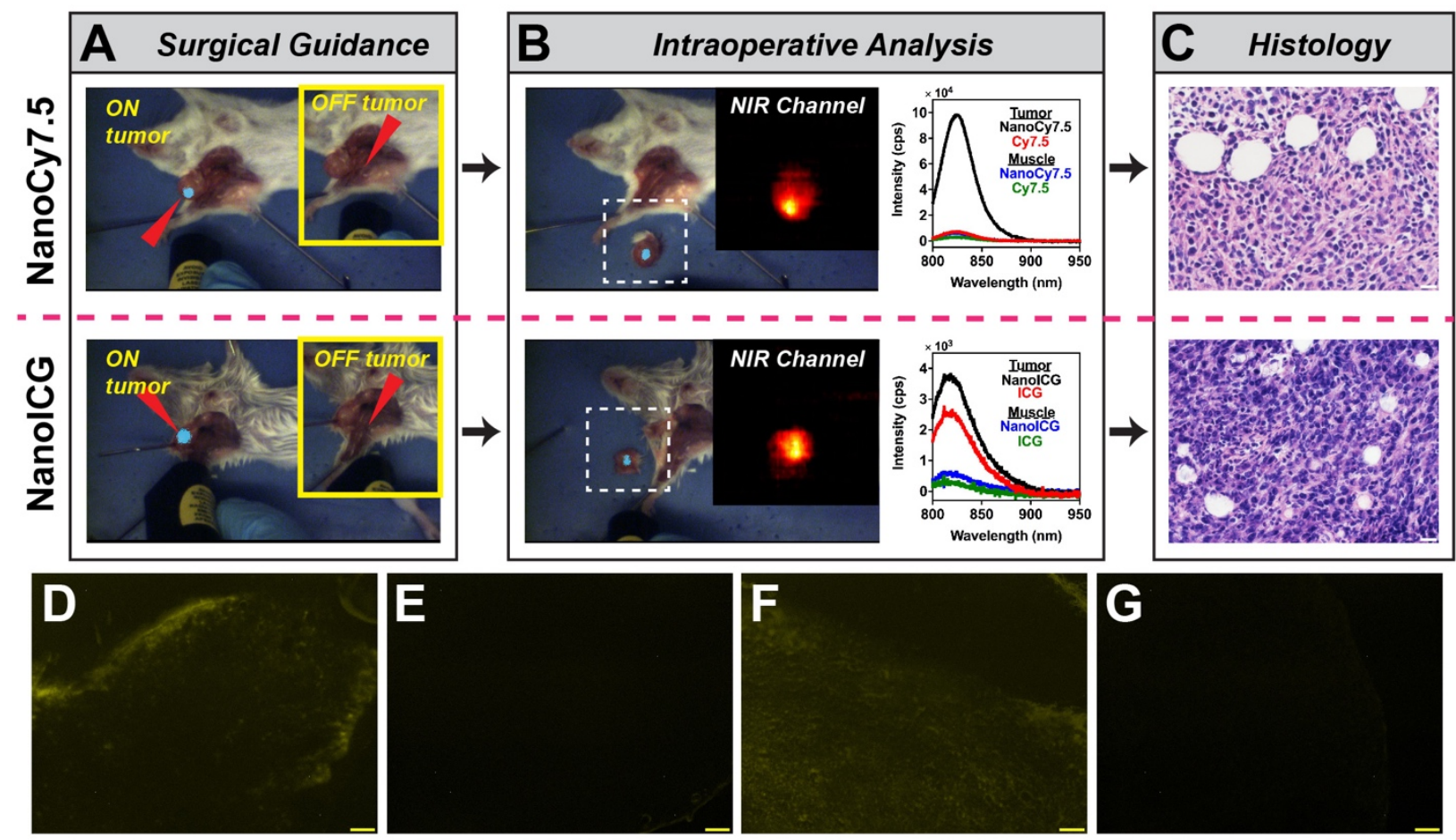

Figure 9. Image-guided surgery in mice bearing orthotopic 4T1 breast tumors. (A) Surgical (in situ) detection of tumor using FIGSS of NanoCy7.5 ${ }_{100-H}$ (upper) and NanolCGpBA (lower). Red arrowheads indicated the directed location of the excitation laser. The main image is when the laser is directed at an enhancing (i.e. tumor) location, while the image inset (yellow box) shows imaging when the laser is directed off tumor. (B) Intraoperative analysis is demonstrated by FIGSS examination of resected, contrast-enhancing tissue (left) and further spectroscopic analysis (right) normalized to $1 \mathrm{~s}$ integration time. For comparison, spectroscopic signals from Cy7.5 and ICG controls are also shown. The inset is the NIR channel of FIGSS zoomed in from the area in the dashed white box. NIR fluorescence in the tumor is observed for both NanoCy7.5100-H and NanoICG pBA. (C) H\&E stained specimens from contrast enhancing regions indicated malignant tissue; scale bar $=20 \mu \mathrm{m}$. (D-G) Representative fluorescence micrographs in the NIR channel after frozen sectioning: (D) NanoCy7.5100-H, (E) Cy7.5, (F) NanolCG 

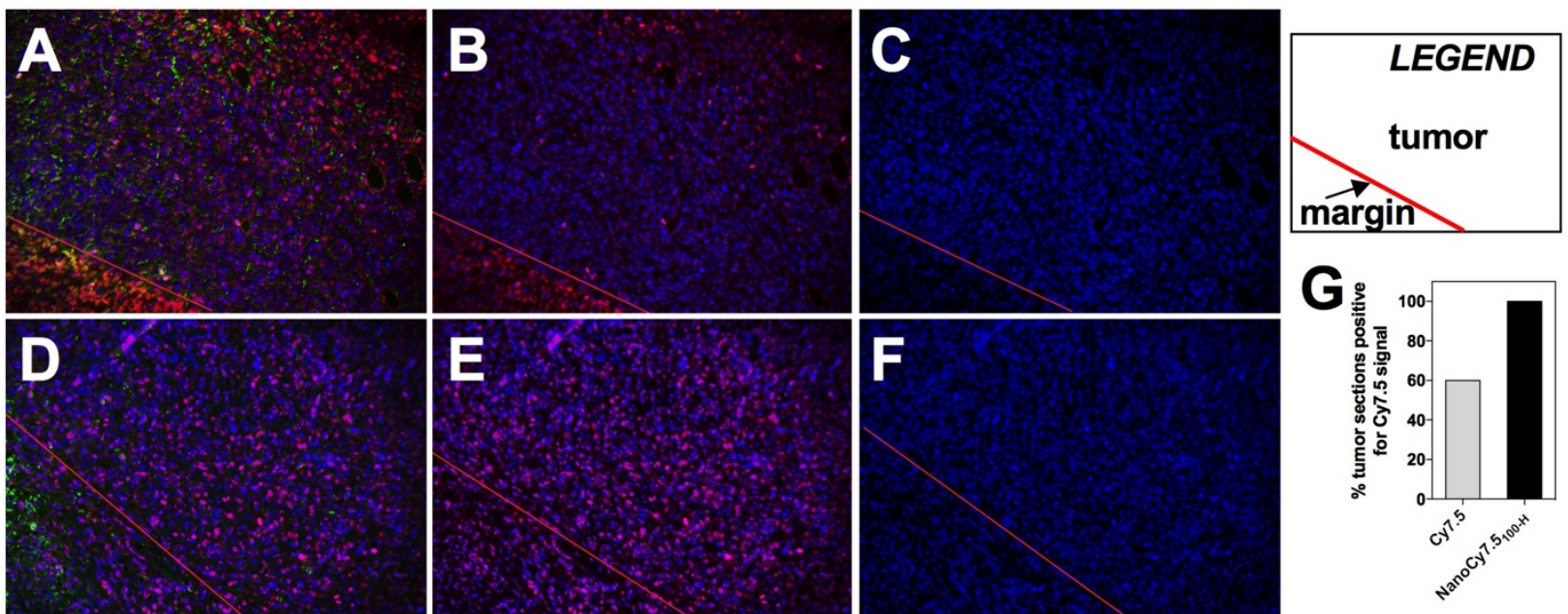

Figure 10. Detection of Cy7.5 and NanoCy7.5100-H in tumor sections. 4T1 tumors from BALB/c mice injected with either Cy7.5 or NanoCy7.5 $100-\mathrm{H}$ after $24 \mathrm{hr}$ exposure were harvested, immediately fixed, and sectioned. Tumor slides where reacted with a multiplexed panel of antibodies, and both the Cy7.5 signal and multiplexed Opal stains were imaged using a Nuance Camera. Spectral unmixing of the multiplexed panel was performed by InForm software (Perkin-Elmer, Hopkington, MA), and quantitation of the fixed Cy7.5 or NanoCy7.5 100-H in the tumor was calculated. Panel (A)-(C) represent tumors exposed to Cy7.5, and panels (D)-(F) are tumors exposed to NanoCy7.5100-H. A red line is provided to delineate the tumor margin. Column 1 is the multiplexed image of DAPI (blue) + dye (Cy7.5 or NanoCy7.5; pseudo-colored magenta) + tumor-pancytokeratin (green), Column 2 represents DAPI (blue) + dye (either Cy7.5 or NanoCy7.5; pseudo-colored magenta), Column 3 represents DAPI alone (blue). Over 800 images taken from more than 19 slides/tumor were analyzed in Inform and NIR signal in tumor was quantified (G).

Histological analysis of BALB/c tumors taken 24 $\mathrm{h}$ after contrast agent injection utilized spectral unmixing and tissue/cell level segmentation to determine areas within slides which were considered tumor (Figure 10A-F). Alpha smooth muscle actin was used as a stromal marker while pan-cytokeratin was used as a tumor marker, followed by training and processing with InForm software. Results showed that tumors from mice treated with Cy7.5 alone (top row) had fewer tumor images that were positive for the NIR fluorescent Cy7.5 signal (Figure 10B), compared to tumors from mice treated with NanoCy7.5100-H (Figure 10F). Interestingly, of the slides examined from resected 4T1 tumors, 9 out of 15 tumor slides $(60 \%)$ from BALB/c mice that were administered Cy7.5 alone showed signal. Conversely, 19 out of 19 slides from tumors imaged with NanoCy7.5100-H showed signal NIR signal (Figure 10G).

\section{Discussion}

We synthesized eight NP formulations derived from HA based on methods that we have previously described [26,27] to comprehensively assess delivery of the NIR fluorophores ICG and Cy7.5 in two models of TNBC. Despite the higher dose of ICG and NanoICG formulations (10 nmol/mouse) compared to Cy7.5 formulations (1.2 nmol/mouse), Cy7.5 formulations showed higher SNR in all tissues through all time points. This resulted, in part, from a higher quantum yield from Cy7.5 compared to ICG, as well as direct conjugation of Cy7.5 to HA. Clearance of ICG and NanoICG also appears to have been more rapid, potentially due to two factors. First, ICG experiences relatively rapid biliary clearance [21], and ICG loaded into NanoICG formulations is not covalently bound. This may allow for gradual release of ICG to serum proteins and hepatobiliary clearance associated with the dye. Conversely, Cy7.5 was directly conjugated to HA through an amide bond, thereby HA governed the clearance Cy7.5. High fluorescence signals in the liver, spleen, and kidney were observed throughout for all contrast agents, consistent with clearance of NPs [39]. NanoCy7.5 formulations also showed initially higher signals in the lungs at $4 \mathrm{~h}$, possibly due to the high degree of vascularization of lung tissue and then decreased at later time points as the polymers cleared from the blood. NanoCy7.5 conjugates also showed somewhat greater retention in the bone, possibly due to HA targeting osteal CD44 expression [40].

Muscle tissue was chosen for contrast since it is highly vascularized, and thus represent signal proportional to that found in blood. Additionally, acquiring sufficient healthy breast tissue from nude mice for contrast was not feasible due to the orthotopic tumor model. As can be seen in Figure 3, CNR values were relatively low at $4 \mathrm{~h}$ and had much higher variability compared to 24 and $72 \mathrm{~h}$. In many cases, individual mice and the groups as a whole had higher signal in muscle tissue than tumor. This is likely the result of a combination of factors. First, NPs and small molecules may not have had sufficient time to extravasate from the tumor vasculature into the tumor cell and stroma in large amounts. In this case, tumor signal would primarily be due to contrast 
agents within circulation. As muscle is generally well supplied with blood, it should have signal similar to that in the tumors. However, as contrast agents are cleared from the blood over 24 and $72 \mathrm{~h}$, the signal in the muscle should decrease proportionally, while little extravasation from the tumor is expected. Thus, tumor signal would be retained to a higher degree in the tumor, resulting in greater contrast at later time points.

The greatest contrast was observed using directly conjugated Cy7.5 to HA. This is likely the result of the higher quantum yield of Cy7.5, longer circulation of Cy7.5 as NP or polymeric conjugate (Supplementary Figure 1), as well as a reduced clearance from the tumors due to the higher MW of the conjugates [39]. Interestingly, the highest contrast enhancement was observed at $72 \mathrm{~h}$ for the $10 \mathrm{kDa}$ conjugates, while the highest contrast at $24 \mathrm{~h}$ was observed with NanoCy7.5100-H. This appears to have resulted from a combination of decreasing muscle signal in mice treated with NanoCy7.510, as well as a tumor clearance of contrast agent between 24 and 72 h. Mice treated with NanoCy7.5 $5_{100-H}$ however showed a substantial decrease in tumor signal between 24 and $72 \mathrm{~h}$. NanoICG formulations provided higher contrast than free ICG, despite ICG being physically entrapped within the NP core rather than directly conjugated, which is also consistent with our previous results [26]. This indicates that while the NP formulation provides a distinct advantage to delivery of the fluorophores. Additionally, ICG is already clinically approved and loading into HA NPs does not require chemical modification, which may offer some advantages.

CNR measures the difference in signal between two tissues and is important for a contrast agent's ability to differentiate tissues of interest. However, it does not directly compare the effectiveness of different nanoparticle formulations. CNR, as defined by, (Tumor SNR - Muscle SNR)/(standard deviation of background), can vary due to quantum yield or dose even though the relative difference between tissues may remain the same. For example, assuming SD of the background is 1, a tumor SNR of 10 and muscle SNR of 5 yields a CNR of 5, while a tumor SNR of 100 and muscle SNR of 50 yields a CNR of 50, despite the dye being distributed proportionally equal. Thus, normalizing tumor SNR from NP contrast agents to their respective free dyes $\left(\mathrm{SNR}_{\mathrm{NP}} / \mathrm{SNR}_{\text {dye }}\right)$ is necessary to compare the ability of $\mathrm{NP}$ formulations to increase tumor signal relative to free dye. NanoCy7.5 formulations showed greater signal in the tumors at each time point with each formulation. At $4 \mathrm{~h}$, NanoCy7.510-H and NanoCy7.5100-L were approximately five times brighter than Cy7.5-amine. NanoCy7.5100-H showed the greatest $\mathrm{SNR} \mathrm{NP}_{\mathrm{NP}} / \mathrm{SNR}_{\text {dye }}$ ratio observed throughout the experiment, being $8.7 \pm 6.0$ times brighter than Cy7.5-amine at $24 \mathrm{~h}$. It is interesting to note that NanoCy7.5100-H showed more specific CD44 targeting compared to other HA-based NPs, especially $10 \mathrm{kDa}$ HA NPs using an in vitro inhibition assay in MDA-MB-231 cells [27], indicating that CD44-HA interaction could influence the significantly higher accumulation in MDA-MB-231 xenografts. Levels for NanoICG were lower than free ICG at $4 \mathrm{~h}$, but 2-3 times higher at 24 and $72 \mathrm{~h}$. These values indicate that the NP formulations substantially improve both signal in the tumor and contrast between other tissues compared to their respective free dyes.

The potential for detecting diseased tissue underlying healthy tissue is an important attribute of NIR fluorescence imaging. It is reported that the maximum practical detection for NIR imaging is around $10 \mathrm{~mm}$ [9]. However, this detection limit is dependent on the flux of the excitation light and the quantity of fluorophore that has accumulated in the diseased tissue compared to surrounding tissue. In previous studies, using an identical image-guided surgery system, we were able to observe ICG fluorescence spectra at approximately $5-7 \mathrm{~mm}$ depending on the tissue using $785 \mathrm{~nm}$ laser, $200 \mathrm{~mW}$ power [35]. In comparison, this study observed ICG and Cy7.5 signal up to 3 and $6 \mathrm{~mm}$ deep using $30 \mathrm{~mW}$ laser power. Most contrast agents examined in this study were detectable to no more than $4 \mathrm{~mm}$ deep, and this limit is likely to be lower when additional contrast agent is present in the intervening tissue. However, this still represents a substantial improvement over imaging in the visible range which limits detection depth to only a few micrometers. Future studies will utilize a series of phantoms containing different levels of contrast agent approximating different tissues likely to be encountered during surgery.

NanoCy7.5100-H and NanoICG lead compounds for their improved CNRs at $24 \mathrm{~h}$. Biodistribution was similar between the BALB/c and nude mice. Though nude mice lack functional T-cells, it was unknown whether the fully functional immune system the BALB/c mice would significantly alter relative biodistribution or clearance as both models have functional innate immunity. Contrast-enhancement results were similar between nude and BALB/C mice as well, with ICG having greater contrast than NanoICGPBA at $4 \mathrm{~h}$, while NanoICGPBA had greater contrast at $24 \mathrm{~h}$. NanoCy7.5 $1_{100-\mathrm{H}}$ exhibited greater contrast at both 4 and $24 \mathrm{~h}$, similar to results in nude mice. The contrast levels of all contrast agents, however, were 
significantly higher in the BALB/c model than the nude mouse model, with CNR for NanoICGPBA as high as $27.0 \pm 3.0$, and CNR for NanoCy7.5100-H as high as $245.7 \pm 78.5$, compared to $13.8 \pm 4.6$ and $101.9 \pm 52.9$ for their respective values in nude mice at $24 \mathrm{~h}$. Similarly, $\mathrm{SNR}_{\mathrm{NP}} / \mathrm{SNR}_{\text {dye }}$ ratios were increased in these models as well. This is likely a result of the tumor model used in each instance. MDA-MB-231 tumors were smaller and slower growing compared to highly aggressive, rapidly growing 4T1 tumors. This increased growth rate may be representative of a greater vascular supply in the 4T1 tumors, which could provide a greater potential for NP delivery.

Histological analysis of tumors taken from BALB/c mice at 24 treated with either Cy7.5 alone or NanoCy7.5100-H showed a greater fraction of tumor tissue with NIR signal in the NP group compared to dye alone. This could indicate that the NanoCy7.5100-H tumors had higher overall SNR due to increased penetration or retention within the entire tumor compared to Cy7.5 alone, which failed to label certain areas. Additionally, this also indicates that NanoCy7.5100-H more completely identified tumor tissue than did Cy7.5 alone, which may have larger implications for the complete removal of tumor when guided by NIR signal. Future studies will require analysis of NIR signal correlation with other markers such as CD44 and tumor associated fibroblasts, macrophages, and endothelium to determine the precise factors governing the significantly higher signal from HA-based NIRF NPs compared to dye alone in the tumor microenvironment.

\section{Conclusion}

We have demonstrated the efficacy of a panel of NIRF NPs for image-guided surgery in two in vivo TNBC models. Imaging of mice bearing orthotopic breast tumors with NPs using either covalent conjugation (NanoCy7.5) or physicochemical entrapment (NanoICG) resulted in greater tumor signal and contrast than treatment with dye alone, especially at $24 \mathrm{~h}$. The imaging results not only indicated the potential for improved ability to differentiate healthy and diseased tissue, but also provided greater detection through tissue breast tissue mimics suggesting a greater level of potential detection of PMs and local metastatic lesions in the operating room. We also found that chemical conjugation to the HA polymers resulted in greater relative tumor signal compared to dye alone than did physicochemical entrapment, indicating that either passive accumulation of the larger MW polymers, or active targeting to HA-binding moieties assisted in contrast agent uptake and retention. Future studies are necessary to examine the effect on survival after tumor removal using these NP contrast agents and the factors controlling increased tumor contrast and signal retention.

\section{Supplementary Material}

Supplementary tables and figures. http://www.thno.org/v06p2314s1.pdf

\section{Abbreviations}

PM: positive margins; NIR: near-infrared; NIRF: near-infrared fluorescence; NP: nanoparticle; HA: hyaluronic acid; ICG: indocyanine green; IGS: image-guided surgery; EPR: enhanced permeability and retention; SLN: sentinel lymph node; TNBC: triple negative breast cancer; EDC: 1-ethyl-3-(3-dimethylaminopropyl) carbodiimide; NHS: N-hydroxysuccinimide; DMSO: dimethyl sulfoxide; DMF: dimethyl formamide; PBA: aminopropyl-1-pyrenebutanamide; $5 \beta C A$ : aminopropyl-5 $\beta$ cholanamide; SNR: signal to noise ratio; CNR: contrast to noise ratio; DF: detection factor

\section{Acknowledgements}

This work was supported in part by the National Institutes of Health grants R00 CA153916, R01 EB019449, NIH Shared Instrumentation Grant 1S10RR17846 and 1S10RR027940, and the Fred and Pamela Buffett Cancer Center at UNMC (P30 CA036727). TKH thanks the Mike and Lucy Robbins Fellowship for stipend support. We thank Ms. Bowen Qi for technical assistance on animal dissection.

\section{Competing Interests}

The authors have declared that no competing interest exists.

\section{References}

1. DeSantis CE, Lin CC, Mariotto AB, Siegel RL, Stein KD, Kramer JL, Alteri R, Robbins AS, Jemal A. Cancer treatment and survivorship statistics, 2014. CA Cancer J Clin. 2014; 64(4):252-71.

2. Pleijhuis RG, Graafland M, de Vries J, Bart J, de Jong JS, van Dam GM. Obtaining adequate surgical margins in breast-conserving therapy for patients with early-stage breast cancer: current modalities and future directions. Ann Surg Oncol. 2009; 16(10):2717-30.

3. Rizzo M, Iyengar R, Gabram SG a, Park J, Birdsong G, Chandler KL, Mosunjac MB. The effects of additional tumor cavity sampling at the time of breast-conserving surgery on final margin status, volume of resection, and pathologist workload. Ann Surg Oncol. 2010;17(1):228-34.

4. Meric F, Mirza NQ, Vlastos G, Buchholz TA, Kuerer HM, Babiera G V, Singletary SE, Ross MI, Ames FC, Feig BW, Krishnamurthy S, Perkins GH, McNeese MD, Strom EA, Valero V, Hunt KK. Positive surgical margins and ipsilateral breast tumor recurrence predict disease-specific survival after breast-conserving therapy. Cancer. 2003; 97(4):926-33.

5. Wapnir IL, Anderson SJ, Mamounas EP, Geyer CE, Jeong J-H, Tan-Chiu E, Fisher B, Wolmark N. Prognosis after ipsilateral breast tumor recurrence and locoregional recurrences in five National Surgical Adjuvant Breast and Bowel Project node-positive adjuvant breast cancer trials. J Clin Oncol. 2006; 24(13):2028-37.

6. Komoike Y, Akiyama F, lino Y, Ikeda T, Akashi-Tanaka S, Ohsumi S, Kusama M, Sano M, Shin E, Suemasu K, Sonoo H, Taguchi T, Nishi T, Nishimura R, Haga S, Mise K, Kinoshita T, Murakami S, Yoshimoto M, Tsukuma H, Inaji H. Ipsilateral breast tumor recurrence (IBTR) after breast-conserving treatment for early breast cancer: Risk factors and impact on distant metastases. Cancer. 2006;106(1):35-41. 
7. Frangioni J V. New technologies for human cancer imaging. J Clin Oncol. 2008; 26(24):4012-21.

8. Lovrics PJ, Cornacchi SD, Vora R, Goldsmith CH, Kahnamoui K. Systematic review of radioguided surgery for non-palpable breast cancer. Eur J Surg Oncol. 2011;37(5):388-97.

9. Vahrmeijer AL, Hutteman M, van der Vorst JR, van de Velde CJH, Frangioni J $\mathrm{V}$. Image-guided cancer surgery using near-infrared fluorescence. Nat Rev Clin Oncol. 2013;10(9):507-18.

10. Rosenthal EL, Warram JM, Bland KI, Zinn KR. The Status of Contemporary Image-Guided Modalities in Oncologic Surgery. Ann Surg. 2015;261:46-55.

11. Behbahaninia M, Martirosyan NL, Georges J, Udovich JA, Kalani MYS, Feuerstein BG, Nakaji P, Spetzler RF, Preul MC. Intraoperative fluorescent imaging of intracranial tumors: A review. Clin Neurol Neurosurg. 2013;115(5):517-28.

12. Sevick-Muraca EM. Translation of near-infrared fluorescence imaging technologies: emerging clinical applications. Annu Rev Med. 2012;63:217-31.

13. Fass L. Imaging and cancer: a review. Mol Oncol. 2008;2(2):115-52.

14. Richards-Kortum R, Sevick-Muraca E. Quantitative optical spectroscopy for tissue diagnosis. Annu Rev Phys Chem. 1996;47:555-606.

15. Hill TK, Mohs AM. Image-guided tumor surgery: Will there be a role for fluorescent nanoparticles? WIREs Nanomed Nanobiotechnol. 2015.

16. van Dam GM, Themelis G, Crane LMA, Harlaar NJ, Pleijhuis RG, Kelder W, Sarantopoulos A, de Jong JS, Arts HJG, van der Zee AGJ, Bart J, Low PS, Ntziachristos V. Intraoperative tumor-specific fluorescence imaging in ovarian cancer by folate receptor- $a$ targeting: first in-human results. Nat Med. 2011;1-6

17. van der Vorst JR, Schaafsma BE, Verbeek FP, Keereweer S, Jansen JC, van der Velden LA, Langeveld AP, Hutteman M, Lowik CW, van de Velde CJ, Frangioni J V, Vahrmeijer AL. Near-infrared fluorescence sentinel lymph node mapping of the oral cavity in head and neck cancer patients. Oral Oncol. 2013;49(1):15-19.

18. Ishizawa $\mathrm{T}$, Fukushima $\mathrm{N}$, Shibahara J, Masuda $\mathrm{K}$, Tamura $\mathrm{S}$, Aoki $\mathrm{T}$, Hasegawa K, Beck Y, Fukayama M, Kokudo N. Real-time identification of liver cancers by using indocyanine green fluorescent imaging. Cancer. 2009:115(11):2491-504.

19. Tummers QRJG, Verbeek FPR, Schaafsma BE, Boonstra MC, van der Vorst JR, Liefers G-J, van de Velde CJH, Frangioni J V, Vahrmeijer AL. Real-time intraoperative detection of breast cancer using near-infrared fluorescence imaging and Methylene Blue. Eur J Surg Oncol. 2014;40(7):850-8.

20. Alander JT, Kaartinen I, Laakso A, Pätilä T, Spillmann T, Tuchin V V, Venermo $\mathrm{M}$, Välisuo P. A review of indocyanine green fluorescent imaging in surgery. Int J Biomed Imaging. 2012;2012:1-27.

21. Schaafsma BE, Mieog JSD, Hutteman M, van der Vorst JR, Kuppen PJK, Löwik CWGM, Frangioni J V, van de Velde $\mathrm{CJH}$, Vahrmeijer AL. The clinical use of indocyanine green as a near-infrared fluorescent contrast agent for image-guided oncologic surgery. J Surg Oncol. 2011;104(3):323-32.

22. van Dam GM. VEGF-targeted Fluorescent Tracer Imaging in Breast Cancer. ClinicalTrials.gov. 2015;NCT0150857.

23. Rosenthal E. Cetuximab IRDye800 Study as an Optical Imaging Agent to Detect Cancer During Surgical Procedures. ClinicalTrials.gov. 2015;NCT0198737.

24. Verbeek FPR, van der Vorst JR, Tummers ORJG, Boonstra MC, de Rooij KE, Löwik CWGM, Valentijn a RPM, van de Velde CJH, Choi HS, Frangioni J V, Vahrmeijer AL. Near-infrared fluorescence imaging of both colorectal cancer and ureters using a low-dose integrin targeted probe. Ann Surg Oncol. 2014;21:528-37.

25. Choi KY, Jeon EJ, Yoon HY, Lee BS, Na JH, Min KH, Kim SY, Myung S-J, Lee S, Chen X, Kwon IC, Choi K, Jeong SY, Kim K, Park JH. Theranostic nanoparticles based on PEGylated hyaluronic acid for the diagnosis, therapy and monitoring of colon cancer. Biomaterials. $2012 ; 33(26): 6186-93$.

26. Hill TK, Abdulahad A, Kelkar SS, Marini FC, Long TE, Provenzale JM, Mohs AM. Indocyanine Green-Loaded Nanoparticles for Image-Guided Tumor Surgery. Bioconjug Chem. 2015;26:294-303.

27. Kelkar SS, Hill TK, Marini FC, Mohs AM. Near infrared fluorescent nanoparticles based on hyaluronic acid: Self-assembly, optical properties, and cell interaction. Acta Biomater. 2016:36:1-10.

28. Laurent T, Fraser J. Hyaluronan. FASEB J. 1992;6:2397-404.

29. Yoon HY, Koo H, Choi KY, Chan Kwon I, Choi K, Park JH, Kim K. Photo-crosslinked hyaluronic acid nanoparticles with improved stability for in vivo tumor-targeted drug delivery. Biomaterials. 2013;34(21):5273-80.

30. Cho H-J, Yoon I-S, Yoon HY, Koo H, Jin Y-J, Ko S-H, Shim J-S, Kim K, Kwon IC, Kim D-D. Polyethylene glycol-conjugated hyaluronic acid-ceramide self-assembled nanoparticles for targeted delivery of doxorubicin. Biomaterials. 2012;33(4):1190-200

31. Foulkes WD, Smith IE, Reis-Filho JS. Triple-Negative Breast Cancer. N Engl J Med. 2010;363(20):1938-48.

32. Kaur P, Nagaraja GM, Zheng H, Gizachew D, Galukande M, Krishnan S, Asea A. A mouse model for triple-negative breast cancer tumor-initiating cells (TNBC-TICs) exhibits similar aggressive phenotype to the human disease. BMC Cancer. 2012;12(120):1-12

33. Doolittle E, Peiris PM, Doron G, Goldberg A, Tucci S, Rao S, Shah S, Sylvestre M, Govender P, Turan O, Lee Z, Schiemann WP, Karathanasis E. Spatiotemporal Targeting of a Dual-Ligand Nanoparticle to Cancer Metastasis. ACS Nano. 2015;9(8):8012-21.
34. Choi KY, Min KH, Na JH, Choi K, Kim K, Park JH, Kwon IC, Jeong SY. Self-assembled hyaluronic acid nanoparticles as a potential drug carrier for cancer therapy: synthesis, characterization, and in vivo biodistribution. J Mater Chem. 2009:19(24):4102.

35. Mohs AM, Mancini MC, Singhal S, Provenzale JM, Leyland-Jones B, Wang MD, Nie S. Hand-held Spectroscopic Device for In Vivo and Intraoperative Tumor Detection: Contrast Enhancement, Detection Sensitivity, and Tissue Penetration. Anal Chem. 2010;82(21):9058-65.

36. Mohs AM, Mancini MC, Provenzale JM, Saba CF, Cornell KK, Howerth EW, Nie S. An Integrated Widefield Imaging and Spectroscopy System for Contrast-Enhanced, Image-guided Resection of Tumors. IEEE Trans Biomed Eng. 2015;62(5):1416-24.

37. De Grand AM, Lomnes SJ, Lee DS, Pietrzykowski M, Ohnishi S, Morgan TG, Gogbashian A, Laurence RG, Frangioni J V. Tissue-like phantoms for near-infrared fluorescence imaging system assessment and the training of surgeons. J Biomed Opt. 2006;11(1):1-10.

38. Pleijhuis RG, Langhout GC, Helfrich W, Themelis G, Sarantopoulos A, Crane L, Harlaar N, de Jong J, Ntziachristos V, van Dam G. Near-infrared fluorescence (NIRF) imaging in breast-conserving surgery: assessing intraoperative techniques in tissue-simulating breast phantoms. Eur J Surg Oncol. 2011;37(1):32-9.

39. Bertrand N, Wu J, Xu X, Kamaly N, Farokhzad OC. Cancer nanotechnology: the impact of passive and active targeting in the era of modern cancer biology. Adv Drug Deliv Rev. 2014;66:2-25.

40. Zöller M. CD44: can a cancer-initiating cell profit from an abundantly expressed molecule? Nat Rev Cancer. 2011;11(4):254-67.

\section{Author Biography}

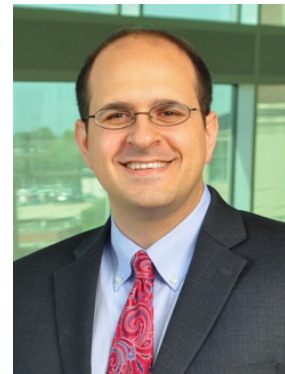

Dr. Aaron M. Mohs is an assistant professor of Pharmaceutical Sciences at the University of Nebraska Medical Center. He is also a member of the Fred and Pamela Buffett Cancer Center and the Center for Drug Delivery and Nanotechnology and the at UNMC. Dr. Mohs has a broad interest in nanotechnology and its use for image-guided procedures, drug delivery, and biosensing. There are three primary projects in the Mohs lab, (1) development on fluorescent nanoparticles for image-guided tumor surgery, (2) nanoparticle formulations of fatty acid synthase inhibitors for tumor therapy, and (3) organic nano-sensors for biochemical sensing. Dr. Mohs has published more than 20 peer-reviewed papers in imaging agent design and development and his research is currently supported by the National Cancer Institute and the National Instutiute of Biomedical Imaging and Bioengineering. Dr. Mohs received his B.A. in Chemistry from St. John's University/College of St. Benedict (MN); Ph.D. in Pharmaceutical Chemistry at the University of Utah and was a postdoctoral fellow in Biomedical Engineering at Emory University. Prior to joining the UNMC, Dr. Mohs was an assistant professor of Biomedical Engineering at the Wake Forest - Virginia Tech School of Biomedical Engineering and Sciences. 